Supporting Information

for

\title{
Supramolecular Self-Assembly Driven Selective Sensing of Phosphates
}

Sahidul Mondal, Tamal Kanti Ghosh, Bijit Chowdhury and Pradyut Ghosh ${ }^{a}$ *

${ }^{a}$ School of Chemical Sciences, Indian Association for the Cultivation of Science, 2A \& 2B Raja

S. C. Mullick Road, Kolkata 700032, India

*Corresponding Author: icpg@iacs.res.in 
1. Figure S1: ${ }^{1} \mathrm{H}-\mathrm{NMR}\left(300 \mathrm{MHz}\right.$ ) spectrum of $\mathbf{L 1}$ in $\mathrm{CDCl}_{3}$

2. Figure S2: ${ }^{13} \mathrm{C}-\mathrm{NMR}(100 \mathrm{MHz})$ spectrum of $\mathbf{L 1}$ in $\mathrm{CDCl}_{3}$

3. Figure S3: ESI-MS spectra of L1.

4. Figure S4: ${ }^{1} \mathrm{H}-\mathrm{NMR}(400 \mathrm{MHz})$ spectrum of Complex $\mathbf{1}\left[\mathbf{P F}_{\mathbf{6}} \mathbf{]}_{\mathbf{2}}\right.$ in Acetone- $d_{6} \quad 5$

5. Figure S5: DEPT-135-NMR $(100 \mathrm{MHz})$ spectrum of $\mathbf{1}\left[\mathbf{P} \mathbf{F}_{\mathbf{6}} \mathbf{]}_{\mathbf{2}}\right.$ in Acetone- $d_{6} \quad 6$

6. Figure S6: ${ }^{13} \mathrm{C}-\mathrm{NMR}(100 \mathrm{MHz})$ spectrum of $\mathbf{1}\left[\mathbf{P F}_{\mathbf{6}}\right]_{2}$ in Acetone- $d_{6} \quad 6$

7. Figure S7: ${ }^{1} \mathrm{H}-{ }^{1} \mathrm{H}$ COSY-NMR $(400 \mathrm{MHz})$ spectrum of $\mathbf{1}\left[\mathbf{P F}_{\mathbf{6}} \mathbf{]}_{2}\right.$ in Acetone- $d_{6} 7$

8. Figure S8: ${ }^{1} \mathrm{H}-{ }^{13} \mathrm{C}$ HMBC-NMR (400 MHz) spectrum of $\mathbf{1}\left[\mathbf{P F}_{\mathbf{6}}\right]_{2}$ in Acetone- $d_{6} 7$

9. Figure S9: ${ }^{1} \mathrm{H}$-DEPT-135 HSQC NMR spectrum of $\mathbf{1}_{\mathbf{1}} \mathbf{P F}_{\mathbf{6}} \mathbf{]}_{\mathbf{2}}$ in Acetone- $d_{6} \quad 8$

10. Figure S10: ESI-MS spectra of $\mathbf{1}\left[\mathrm{PF}_{\mathbf{6}}\right]_{2}$. (a) Isotopic distribution pattern of $\mathbf{1}^{+}$ species with its corresponding simulated pattern

11. Table S1: Crystallographic details of complex $\mathbf{1}\left[\mathbf{P F}_{6}\right]_{2}$ and $\mathbf{1}\left[\mathbf{H}_{2} \mathbf{P O}_{4}\right]_{2}$.

12. Table S2: Selected bond distances and bond angles of complex $\mathbf{1}\left[\mathbf{P F}_{\mathbf{6}} \mathbf{]}_{\mathbf{2}}\right.$.

13. Figure S11: Thermal ellipsoid plot of $\mathbf{1}\left[\mathbf{P F}_{\mathbf{6}} \mathbf{]}_{2}\right.$. (a) Showing C-I $\cdots$ F interaction and (b) showing combined C-I $\cdots \mathrm{F}$ and $\pi-\pi$ stacking interaction. All Ellipsoids are drawn at $30 \%$ probability level.

14. Figure S12: A slice of three-dimensional (3-D) crystal packing of $\mathbf{1}\left[\mathbf{P F}_{\mathbf{6}} \mathbf{l}_{\mathbf{2}}\right.$ along the crystallographic b axis.

15. Figure S13: ${ }^{1} \mathrm{H}-\mathrm{NMR}$ titration profile of $\mathbf{1}\left[\mathbf{P F}_{\mathbf{6}} \mathbf{]}_{\mathbf{2}}\right.$ upon addition of increasing amount of (a) $\mathrm{H}_{2} \mathrm{PO}_{4}^{-}$and (b) $\mathrm{HP}_{2} \mathrm{O}_{7}{ }^{3-}$ (0-2.0 equiv) in DMSO- $d_{6}$.

16. Figure S14: ${ }^{1} \mathrm{H}-\mathrm{NMR}(400 \mathrm{MHz})$ spectrum of $2\left[\mathbf{P F}_{6}\right]_{2}$ in Acetone- $d_{6}$.

17. Figure S15: ${ }^{1} \mathrm{H}-\mathrm{NMR}$ titration profile of $2\left[\mathbf{P F}_{\mathbf{6}}\right]_{2}$ upon addition of increasing amount of (a) $\mathrm{H}_{2} \mathrm{PO}_{4}{ }^{-}$and (b) $\mathrm{HP}_{2} \mathrm{O}_{7}{ }^{3-}$ (0-2.0 equiv) in DMSO- $d_{6}$.

18. Figure S16: ${ }^{1} \mathrm{H}$ DOSY-NMR (500 MHz) spectrum of (a) $\mathbf{1}\left[\mathbf{P F}_{\mathbf{6}} \mathbf{l}_{\mathbf{2}}\right.$; (b) $\mathbf{1}\left[\mathbf{P F}_{\mathbf{6}} \mathbf{l}_{\mathbf{2}}+\mathrm{HP}_{2} \mathrm{O}_{7}{ }^{3-}\right.$ and (c) $\mathbf{1}\left[\mathbf{P F}_{6}\right]_{2}+\mathrm{H}_{2} \mathrm{PO}_{4}^{-}(10 \mathrm{mM})$ in DMSO-d $d_{6}$ at $298 \mathrm{~K}$.

19. Figure S17: ${ }^{1} \mathrm{H}$ DOSY-NMR (500 MHz) spectrum of (a) $\mathbf{1}\left[\mathbf{P F}_{\mathbf{6}}\right]_{2}$, (b) $\mathbf{1}\left[\mathbf{P F}_{\mathbf{6}}\right]_{2}+$ $\mathrm{H}_{2} \mathrm{PO}_{4}{ }^{-}(10 \mu \mathrm{M})$ in DMSO- $d_{6}$ at $298 \mathrm{~K}$ and DLS experiment of (c) $\mathbf{1}\left[\mathbf{P F}_{\mathbf{6}}\right]_{2}$, (d) $\mathbf{1}\left[\mathbf{P F}_{6}\right]_{2}+$ $\mathrm{H}_{2} \mathrm{PO}_{4}^{-}(10 \mu \mathrm{M})$ in DMSO.

20. Table S3: Calculation of sphere volume ratio from DOSY and DLS experiments. 14

21. Figure S18: Anion dependent DLS experiment in DMSO at $298 \mathrm{~K}$.

22. Figure S19: Concentration dependent DLS study of $\mathbf{1}\left[\mathbf{P F}_{\mathbf{6}}\right]_{2}+\mathrm{HP}_{2} \mathrm{O}_{7}{ }^{3-}$ adduct. 15

23. Figure S20: UV and PL plot of $\mathbf{1}\left[\mathbf{P F}_{\mathbf{6}}\right]_{2}$ in acetonitrile at $298 \mathrm{~K} \quad 15$

24. Figure S21: Selectivity Bar-diagram for $\mathbf{1}\left[\mathbf{P F}_{\mathbf{6}} \mathbf{l}_{\mathbf{2}} \quad 15\right.$

25. Figure S22-S23: UV/Vis titration and anion equiv. plot of $\mathbf{1}\left[\mathbf{P F}_{\mathbf{6}}\right]_{2}$ with $\mathrm{H}_{2} \mathrm{PO}_{4}{ }^{-}$/ $\mathrm{HP}_{2} \mathrm{O}_{7}^{3-}$

26. Figure S24-S25: PL titration of $\mathbf{1}\left[\mathbf{P F}_{\mathbf{6}}\right]_{2}$ with $\mathrm{H}_{2} \mathrm{PO}_{4}{ }^{-} / \mathrm{HP}_{2} \mathrm{O}_{7}{ }^{3-} \quad 17$

27. Figure S26: Anion equivalent plot from $\mathrm{PL}$ titration for $\mathbf{1}\left[\mathbf{P F}_{\mathbf{6}} \mathbf{]}_{\mathbf{2}}\right.$ with $\mathrm{H}_{2} \mathrm{PO}_{4}{ }^{-} / \mathrm{HP}_{2} \mathrm{O}_{7}{ }^{3-}$

28. Figure S27: Job's plots from $P L$ for $\mathbf{1}\left[\mathrm{PF}_{\mathbf{6}}\right]_{2}$

29. Figure S28-S29: Non-linear fitting of $\mathrm{PL}$ titration data for $\mathbf{1}\left[\mathbf{P F}_{\mathbf{6}}\right]_{\mathbf{2}}$ with $\mathrm{H}_{2} \mathrm{PO}_{4}^{-} / \mathrm{HP}_{2} \mathrm{O}_{7}^{3-}$

30. Figure S30-S31: Calibration curve for $\mathrm{H}_{2} \mathrm{PO}_{4}{ }^{-} / \mathrm{HP}_{2} \mathrm{O}_{7}{ }^{3-}$ with $\mathbf{1}\left[\mathbf{P F}_{\mathbf{6}}\right]_{2}$ to calculate detection limit

31. Figure S32: Changes in decay pattern of $\mathbf{1}\left[\mathbf{P F}_{\mathbf{6}}\right]_{\mathbf{2}}(10 \mu \mathrm{M})$ and upon addition of one eqv. of $\mathrm{H}_{2} \mathrm{PO}_{4}{ }^{-}$and $\mathrm{HP}_{2} \mathrm{O}_{7}{ }^{3-}$.

32. Figure S33: FESEM images of $\mathbf{1}\left[\mathbf{P F}_{6}\right]_{2}$ and its phosphate adduct. 21

33. Table S4: Selected bond distances and bond angles of complex $\mathbf{1}\left[\mathbf{H}_{\mathbf{2}} \mathbf{P O}_{\mathbf{4}}\right]_{2}$. 
34. Figure S34: Thermal ellipsoid plot of $\mathbf{1}\left[\mathbf{H}_{2} \mathbf{P O}_{4}\right]_{2}$. (a) Showing C-I $\cdots O$ interaction and (b) showing combined C-I $\cdots \mathrm{O}$ and $\pi-\pi$ stacking interaction. All Ellipsoids are drawn at $30 \%$ probability level.

35. Figure S35: (a) Single-crystal X-ray structures of $\mathbf{1}\left[\mathbf{H}_{2} \mathbf{P O}_{4}\right]_{2}$ propagated through combined halogen bonding and $\pi-\pi$ stacking interaction. (b) A slice of threedimensional (3-D) crystal packing of $\mathbf{1}\left[\mathbf{H}_{2} \mathbf{P O}_{4}\right]_{2}$ along the crystallographic a* axis.23 


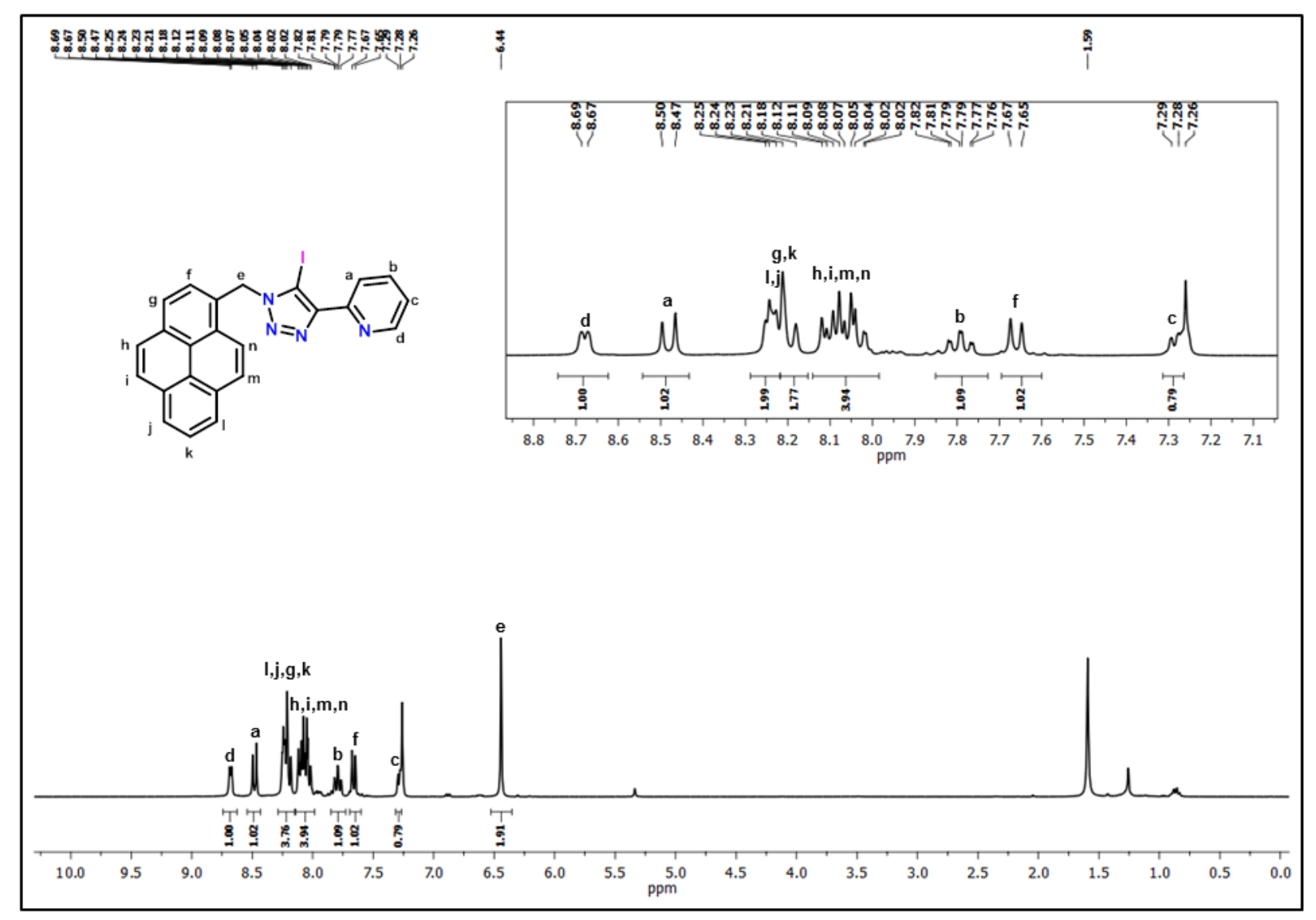

Figure S1: ${ }^{1} \mathrm{H}-\mathrm{NMR}\left(300 \mathrm{MHz}\right.$ ) spectrum of $\mathbf{L 1}$ in $\mathrm{CDCl}_{3}$

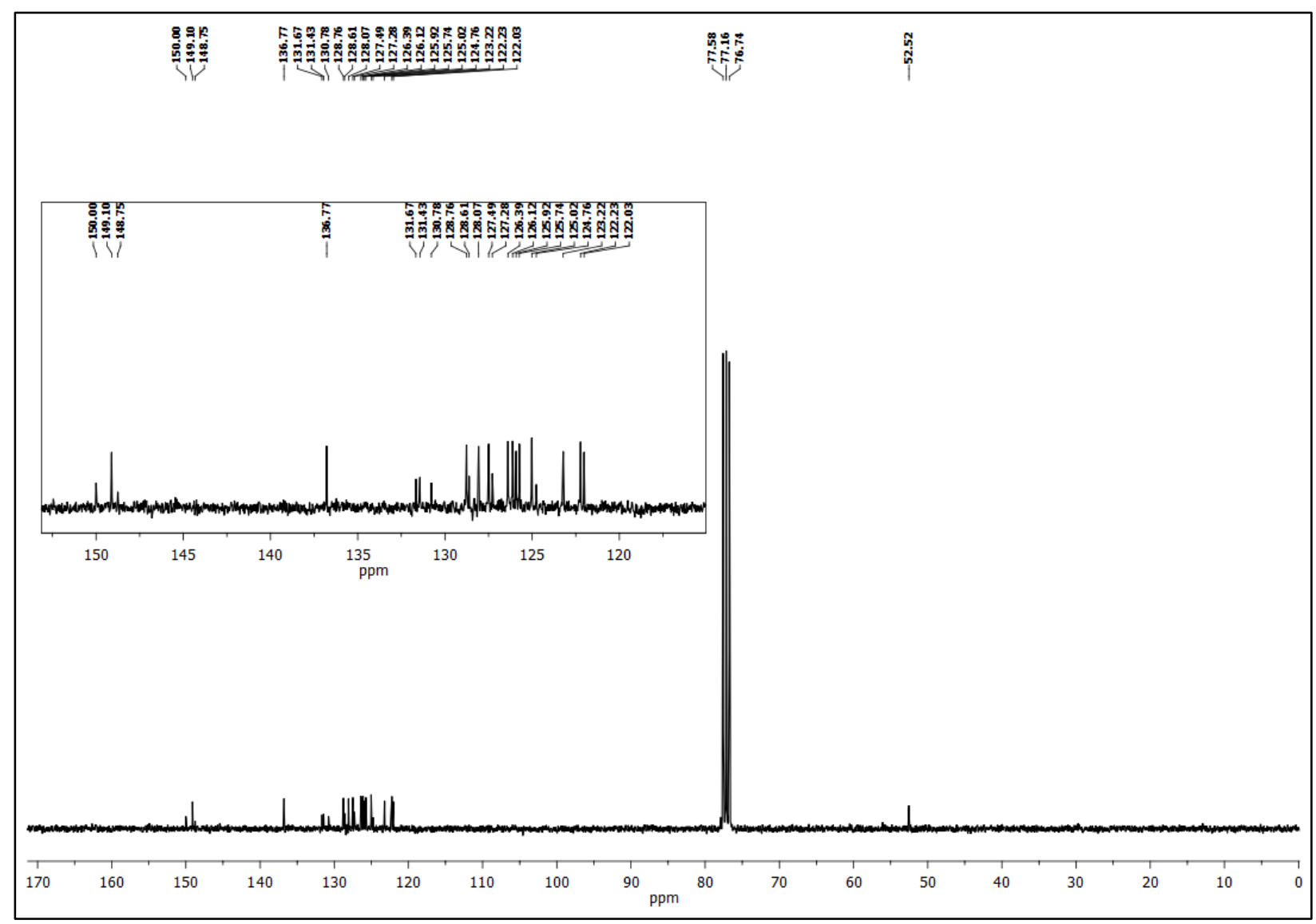

Figure S2: ${ }^{13} \mathrm{C}-\mathrm{NMR}(100 \mathrm{MHz})$ spectrum of $\mathbf{L 1}$ in $\mathrm{CDCl}_{3}$ 


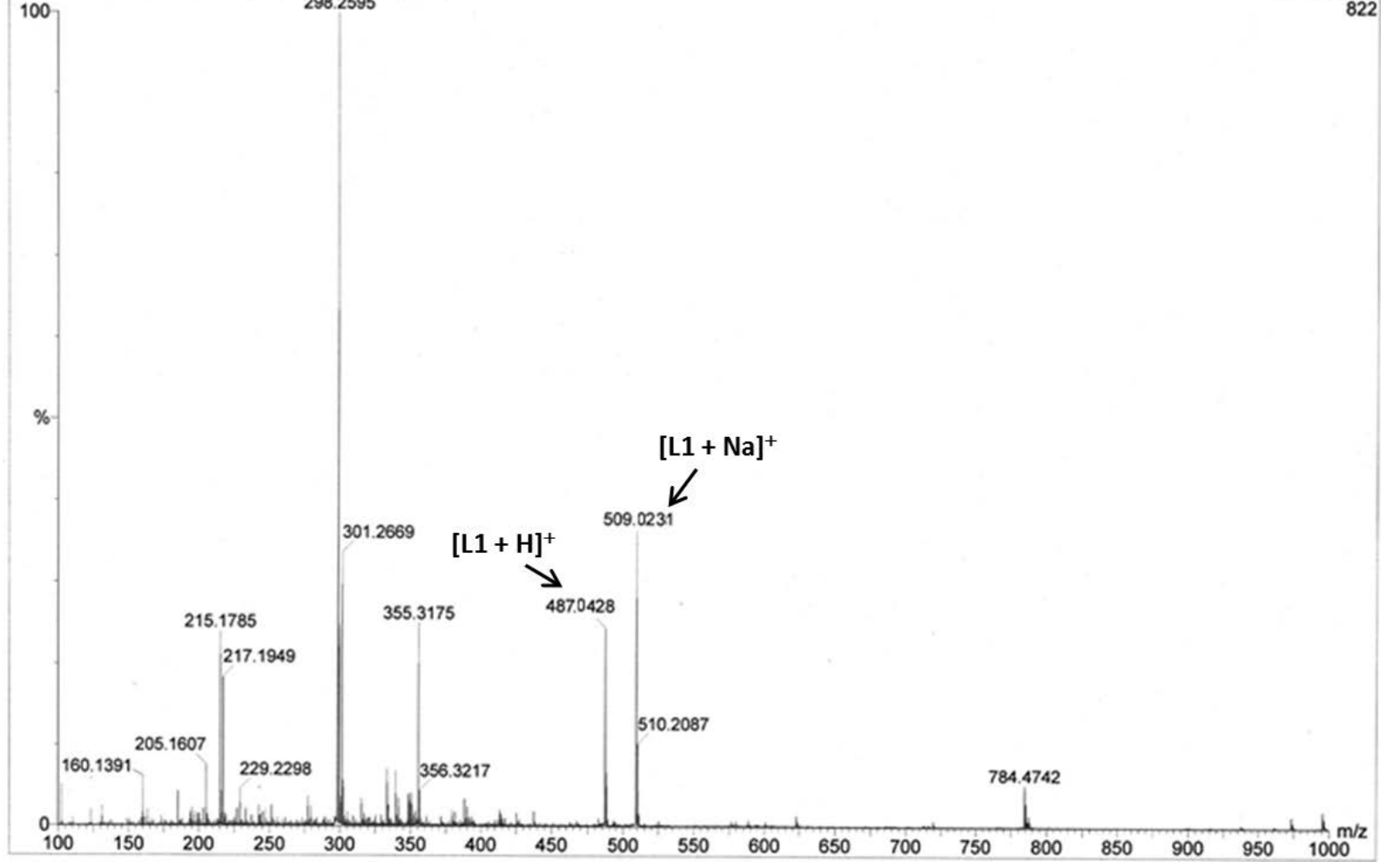

Figure S3: ESI-MS spectra of $\mathbf{L 1}$.

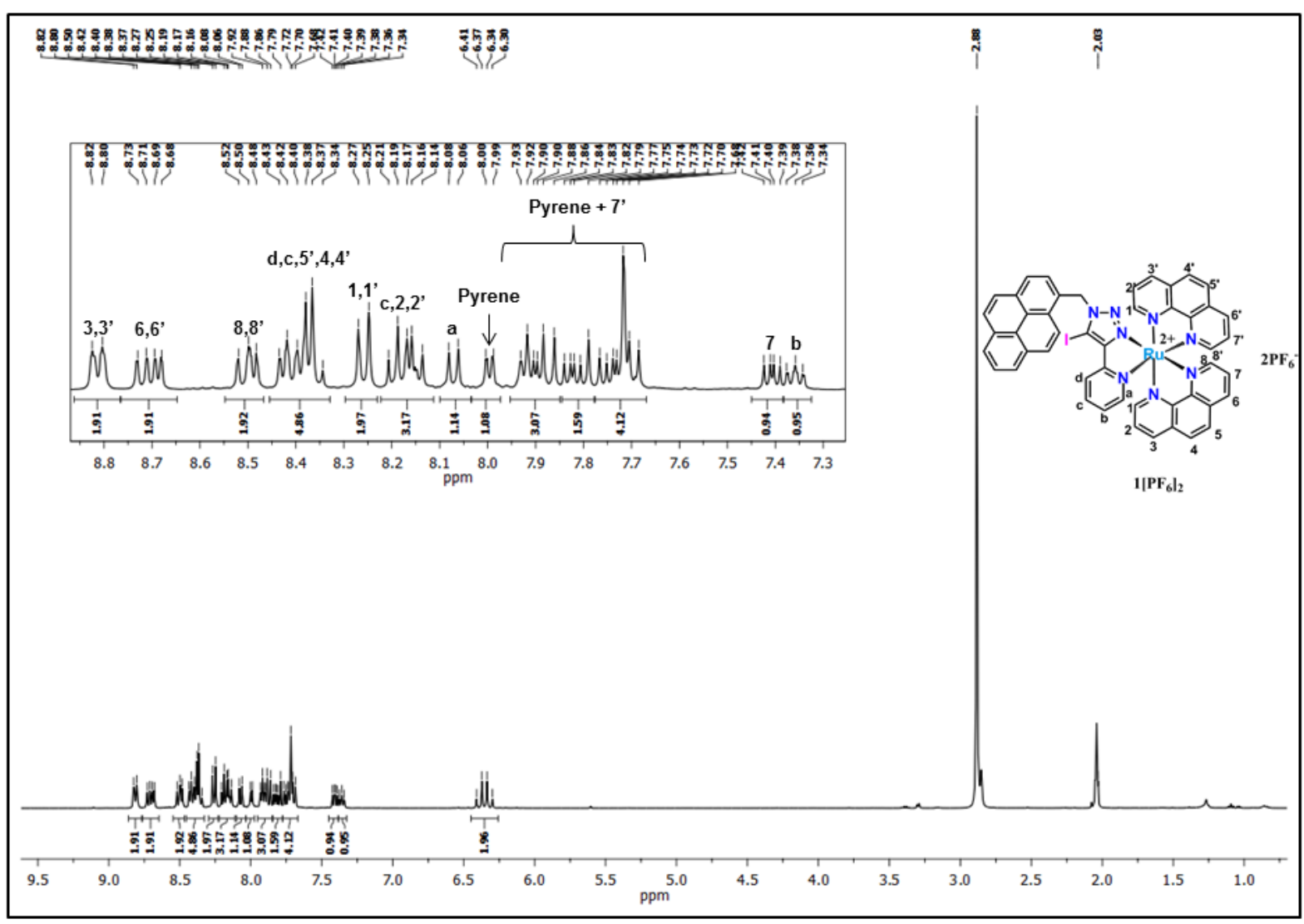

Figure S4: ${ }^{1} \mathrm{H}-\mathrm{NMR}(400 \mathrm{MHz})$ spectrum of Complex $\mathbf{1}\left[\mathbf{P F}_{\mathbf{6}}\right]_{\mathbf{2}}$ in Acetone- $d_{6}$ 


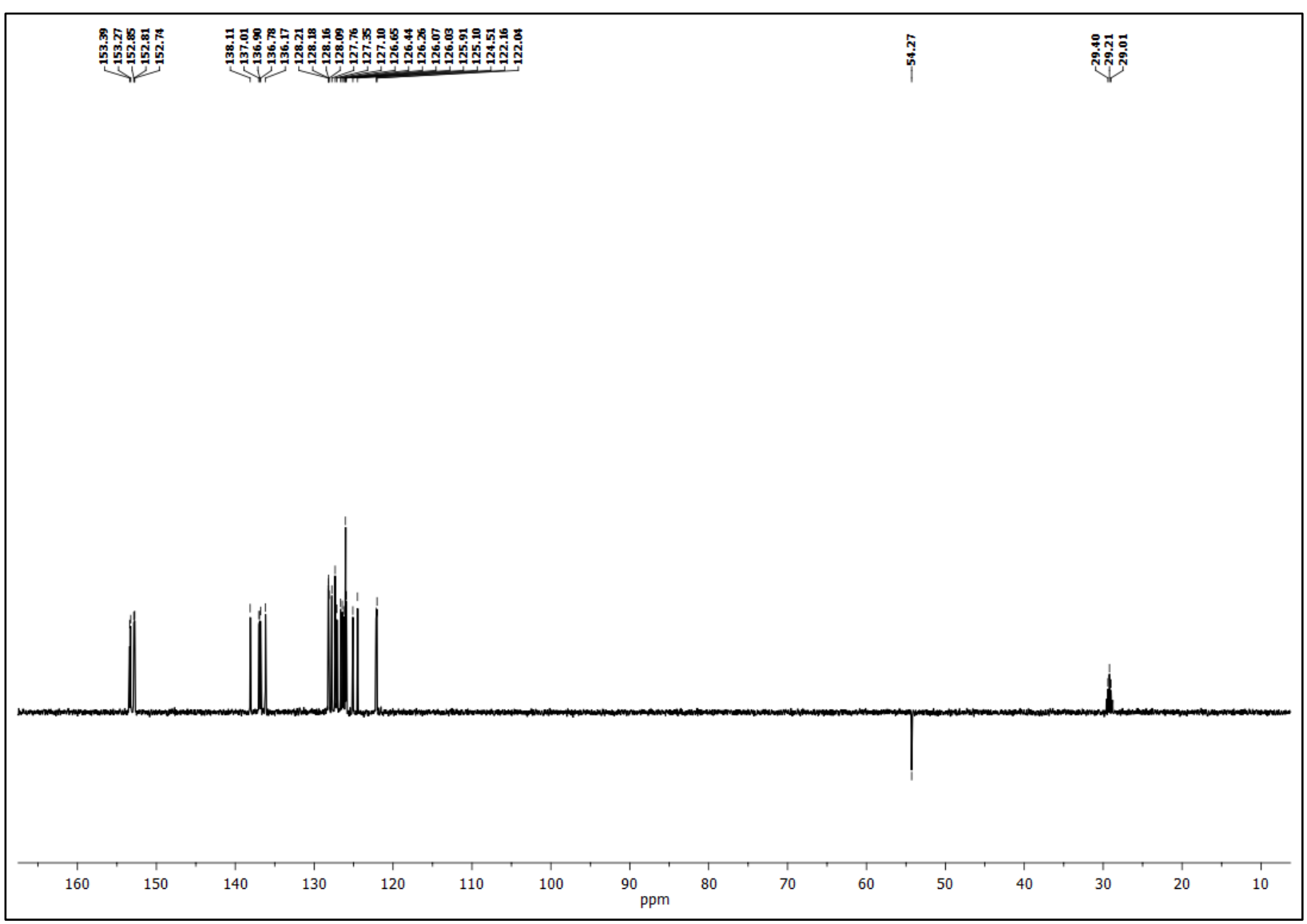

Figure S5: DEPT-135 NMR (100 MHz) spectrum of complex $\mathbf{1}\left[\mathbf{P F}_{\mathbf{6}} \mathbf{]}_{\mathbf{2}}\right.$ in Acetone- $d_{6}$

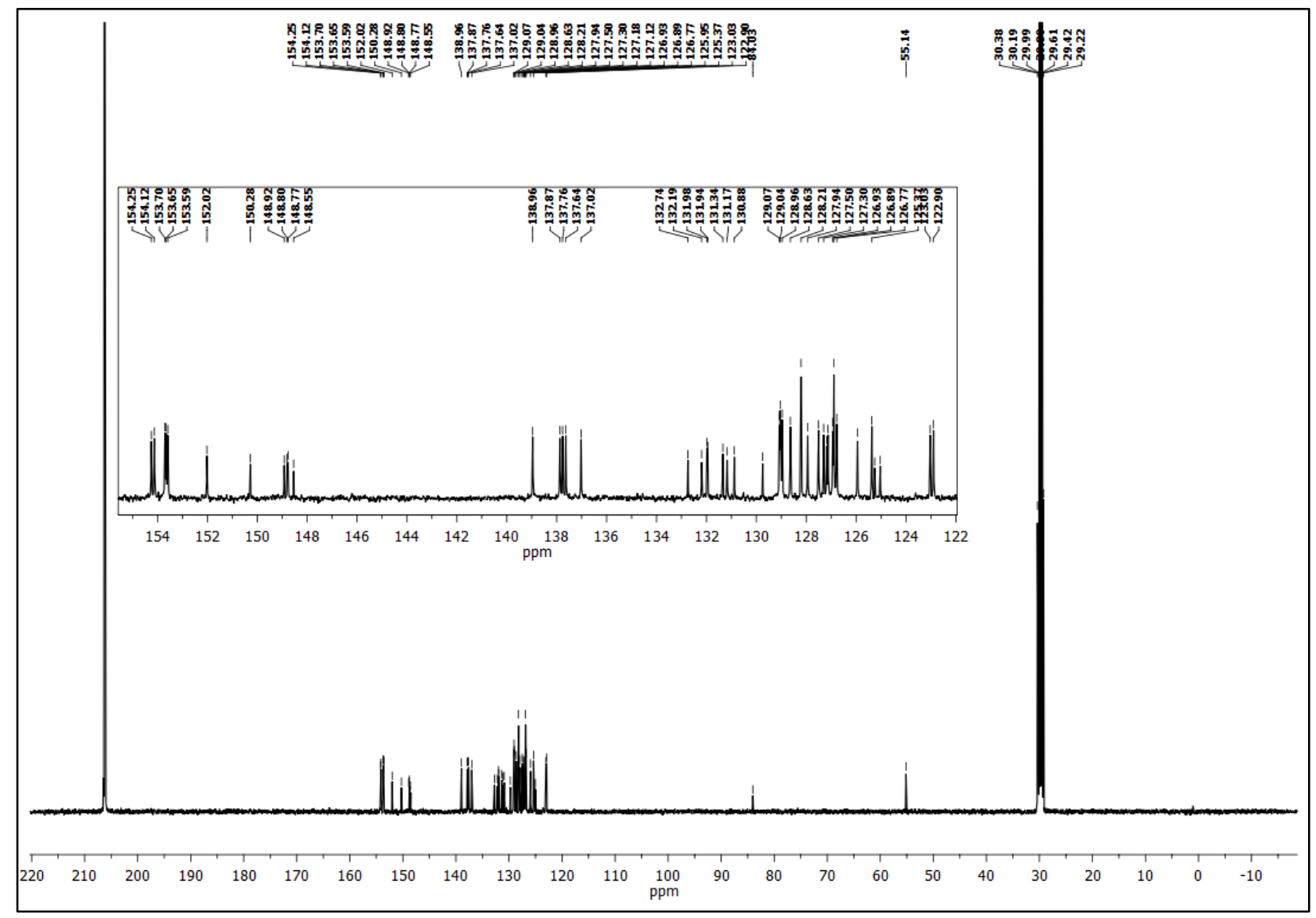

Figure S6: ${ }^{13} \mathrm{C}-\mathrm{NMR}(100 \mathrm{MHz})$ spectrum of complex $\mathbf{1}\left[\mathbf{P F}_{\mathbf{6}} \mathbf{]}_{\mathbf{2}}\right.$ in Acetone- $d_{6}$ 


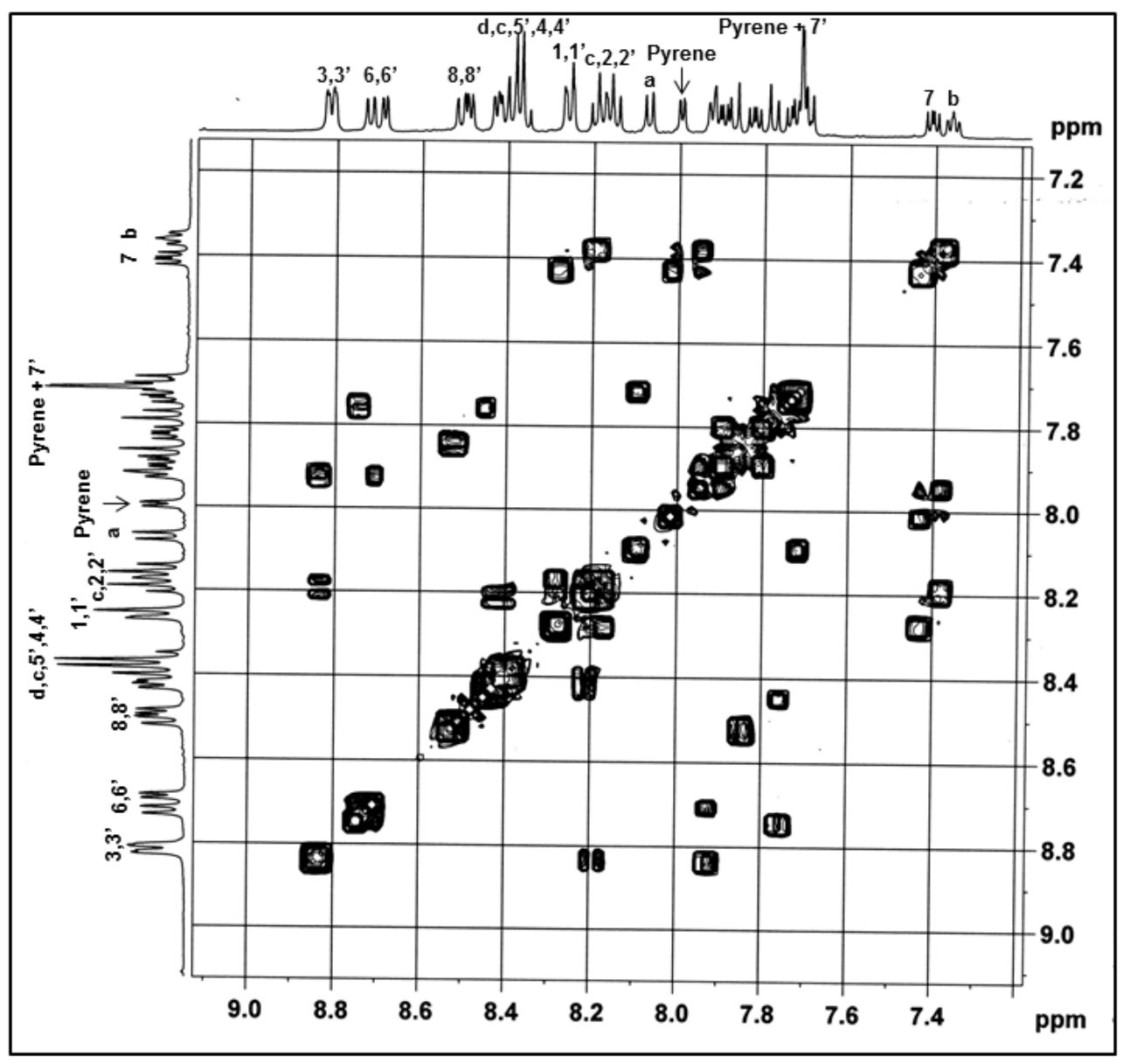

Figure S7: ${ }^{1} \mathrm{H}-{ }^{1} \mathrm{H}$ COSY-NMR (400 MHz) spectrum of complex $\mathbf{1}\left[\mathbf{P F}_{\mathbf{6}} \mathbf{l}_{\mathbf{2}}\right.$ in Acetone- $d_{6}$.

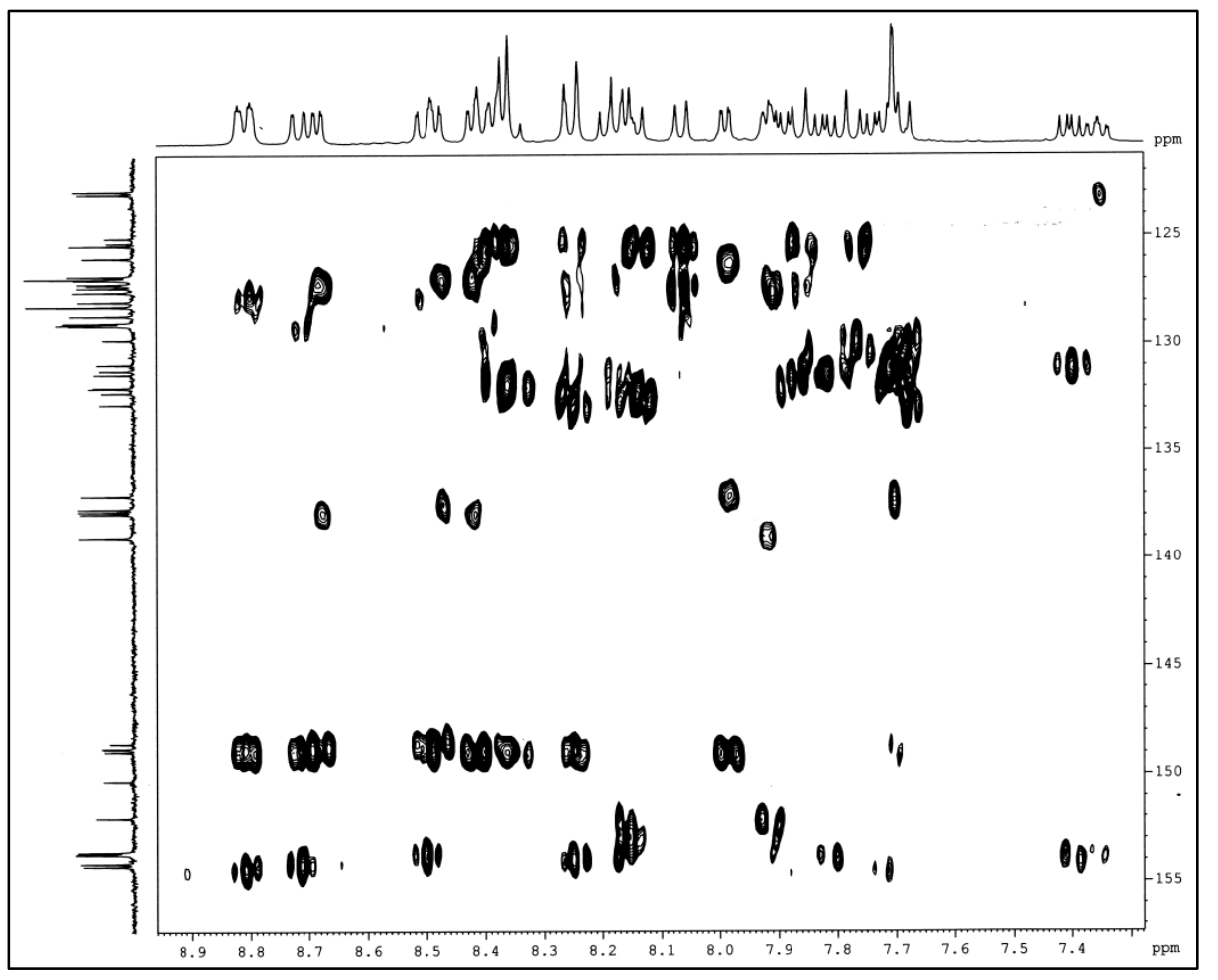

Figure S8: ${ }^{1} \mathrm{H}_{-}{ }^{13} \mathrm{C}$ HMBC-NMR (400 MHz) spectrum of complex $\mathbf{1}\left[\mathbf{P F}_{\mathbf{6}}\right]_{2}$ in Acetone- $d_{6}$. 


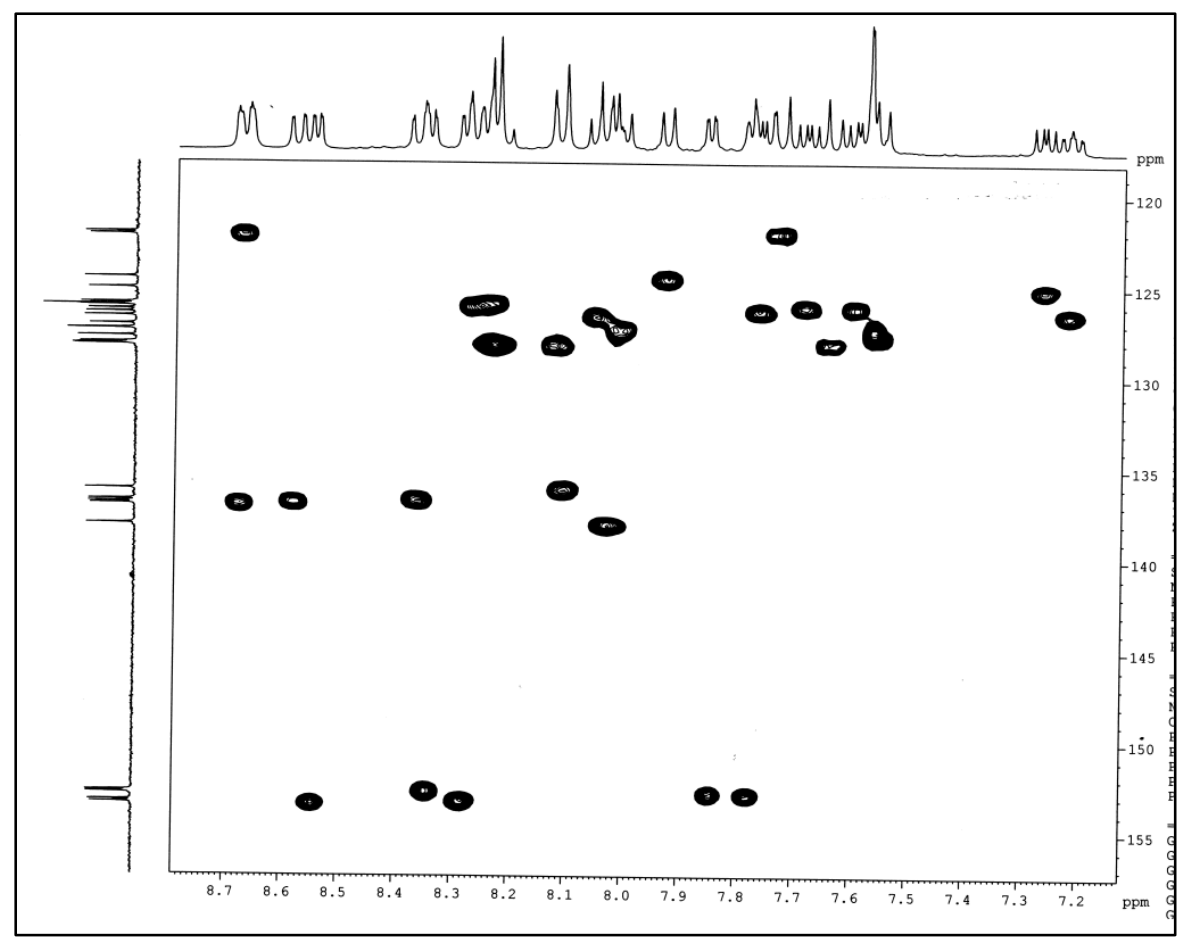

Figure S9: ${ }^{1} \mathrm{H}-\mathrm{DEPT}-135 \mathrm{HSQC}-\mathrm{NMR}$ (400 MHz) spectrum of complex $\mathbf{1}\left[\mathbf{P F}_{\mathbf{6}} \mathbf{]}_{\mathbf{2}}\right.$ in Acetone- $d_{6}$

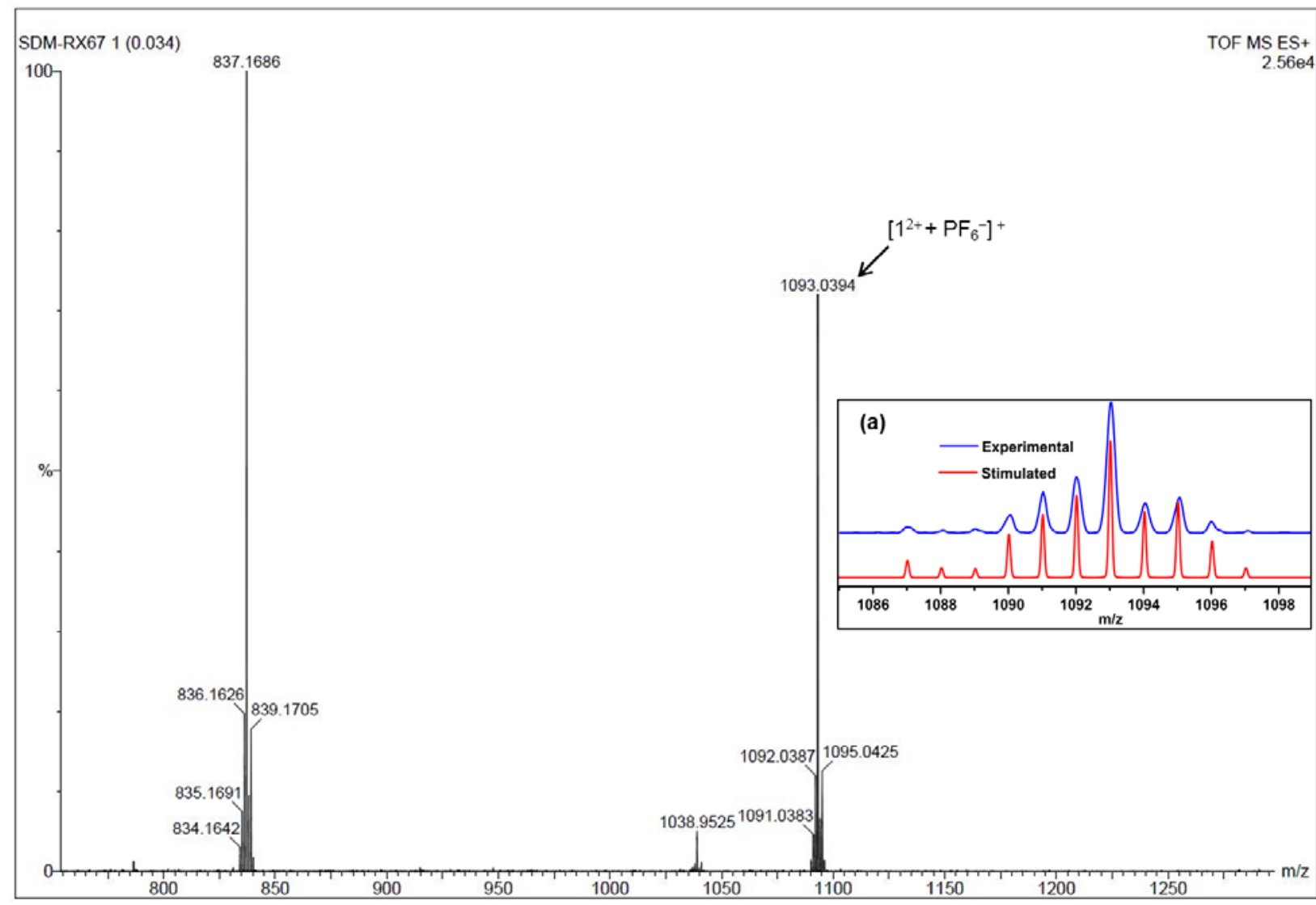

Figure S10: ESI-MS spectra of $\mathbf{1}\left[\mathbf{P F}_{\mathbf{6}}\right]_{2}$. (a) Isotopic distribution pattern of $\mathbf{1}^{+}$species with its corresponding simulated pattern. 
Table S1: Crystallographic details of complex $\mathbf{1}\left[\mathbf{P F}_{6}\right]_{2}$ and $\mathbf{1}\left[\mathbf{H}_{2} \mathbf{P O}_{4}\right]_{2}$.

\begin{tabular}{|c|c|c|}
\hline Compound reference & $\mathbf{1}\left[\mathrm{PF}_{6}\right]_{2}$ & $1\left[\mathrm{H}_{2} \mathrm{PO}_{4}\right]_{2}$ \\
\hline Chemical formula & $\begin{array}{l}\mathrm{C}_{96} \mathrm{H}_{62} \mathrm{I}_{2} \mathrm{~N}_{16} \mathrm{Ru}_{2} \bullet 4\left(\mathrm{PF}_{6}\right) \\
\cdot 6\left(\mathrm{CH}_{2} \mathrm{Cl}_{2}\right)\end{array}$ & $\begin{array}{l}\mathrm{C}_{48} \mathrm{H}_{31} \mathrm{IN} \mathrm{N}_{8} \mathrm{Ru} \cdot 3\left(\mathrm{H}_{2} \mathrm{PO}_{4}\right) \\
\cdot 2\left(\mathrm{H}_{2} \mathrm{O}\right) \cdot 2\left(\mathrm{C}_{3} \mathrm{H}_{7} \mathrm{NO}\right) \cdot \mathrm{C}_{2} \\
\mathrm{H}_{8} \mathrm{~N}\end{array}$ \\
\hline Formula Mass & 2982.97 & 1467.05 \\
\hline Crystal system & triclinic & triclinic \\
\hline$a / \AA ̊ \AA$ & $14.3849(14)$ & $8.2522(19)$ \\
\hline$b / \AA$ & 17.9603(17) & $17.397(4)$ \\
\hline$c / \AA ̊$ & $23.323(2)$ & $23.514(5)$ \\
\hline$\alpha /^{\circ}$ & 100.787(3) & $102.578(8)$ \\
\hline$\beta /^{\circ}$ & 103.071(3) & $95.770(9)$ \\
\hline$\gamma /{ }^{\circ}$ & 96.143(3) & $97.417(8)$ \\
\hline Unit cell volume $/ \AA^{3}$ & 5695.3(9) & $3238.2(12)$ \\
\hline Temperature/K & 150(2) & $110(2)$ \\
\hline Space group & $P \overline{1}$ & $P \overline{1}$ \\
\hline No. of formula units per unit cell, $Z$ & 2 & 2 \\
\hline Radiation type & MoK $\alpha$ & $\mathrm{MoK} \alpha$ \\
\hline No. of reflections measured & 68875 & 28424 \\
\hline No. of independent reflections & 20060 & 10716 \\
\hline $\boldsymbol{R}_{\text {int }}$ & 0.1211 & 0.1774 \\
\hline Final $R_{1}$ values $(I>2 \sigma(I))$ & 0.0848 & 0.1138 \\
\hline Final $w R\left(F^{2}\right)$ values $(I>2 \sigma(I))$ & 0.2225 & 0.2674 \\
\hline Final $R_{1}$ values (all data) & 0.1562 & 0.2010 \\
\hline Final $w R\left(F^{2}\right)$ values (all data) & 0.2680 & 0.3237 \\
\hline CCDC number & 1938836 & 1938835 \\
\hline
\end{tabular}


Table S2: Selected bond distances and bond angles of complex $\mathbf{1}\left[\mathbf{P F}_{\mathbf{6}} \mathbf{]}_{2}\right.$.

\begin{tabular}{|c|c|c|c|c|c|}
\hline M-L & d(M-L)Å & L-M-L & $\angle(\mathbf{L}-\mathrm{M}-\mathrm{L})\left[^{\circ}\right]$ & L-M-L & $\angle(\mathrm{L}-\mathrm{M}-\mathrm{L})\left[^{\circ}\right]$ \\
\hline I1-C01F & $2.051(11)$ & N1-Ru1-N4 & 77.8(3) & N9-Ru2-N12 & $77.5(4)$ \\
\hline I2-C01D & $2.048(11)$ & N1-Ru1-N5 & $95.2(4)$ & N9-Ru2-N13 & $94.0(4)$ \\
\hline Ru1-N1 & $2.028(9)$ & N1-Ru1-N6 & 173.2(3) & N9-Ru2-N14 & $172.2(4)$ \\
\hline Ru1-N4 & $2.046(9)$ & N1-Ru1-N7 & $98.0(3)$ & N9-Ru2-N15 & $98.7(3)$ \\
\hline Ru1-N5 & $2.051(9)$ & N1-Ru1-N8 & $87.6(4)$ & N9-Ru2-N16 & $92.9(4)$ \\
\hline Ru1-N6 & $2.043(9)$ & N4-Ru1-N5 & $89.8(4)$ & N12-Ru2-N13 & $90.3(4)$ \\
\hline Ru1-N7 & $2.070(9)$ & N4-Ru1-N6 & $96.8(4)$ & N12-Ru2-N14 & $97.7(4)$ \\
\hline Ru1-N8 & $2.064(10)$ & N4-Ru1-N7 & $172.9(4)$ & N12-Ru2-N15 & $174.7(3)$ \\
\hline Ru2-N9 & $2.036(8)$ & N4-Ru1-N8 & $94.1(4)$ & N12-Ru2-N16 & $96.6(4)$ \\
\hline Ru2-N12 & $2.080(9)$ & N5-Ru1-N6 & $80.6(4)$ & N13-Ru2-N14 & $79.8(4)$ \\
\hline Ru2-N13 & $2.070(9)$ & N5-Ru1-N7 & $96.4(4)$ & N13-Ru2-N15 & $93.6(3)$ \\
\hline Ru2-N14 & $2.071(9)$ & N5-Ru1-N8 & $175.7(4)$ & N13-Ru2-N16 & 171.1(4) \\
\hline Ru2-N15 & $2.053(9)$ & N6-Ru1-N7 & $87.9(4)$ & N14-Ru2-N15 & $86.5(3)$ \\
\hline Ru2-N16 & $2.061(9)$ & N6-Ru1-N8 & $97.0(4)$ & N14-Ru2-N16 & $93.8(4)$ \\
\hline & & N7-Ru1-N8 & $79.9(4)$ & N15-Ru2-N16 & $79.9(3)$ \\
\hline
\end{tabular}

(a)

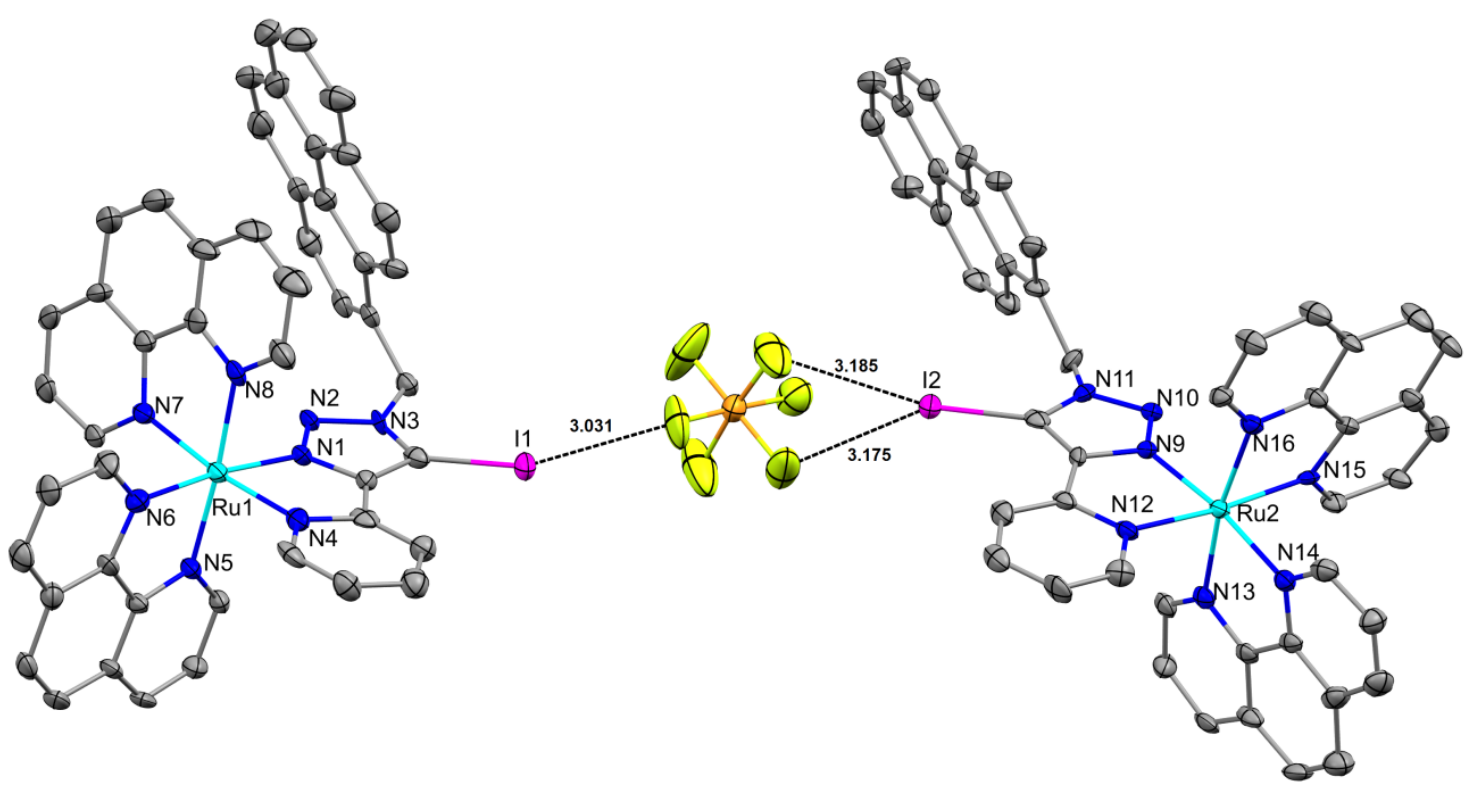


(b)

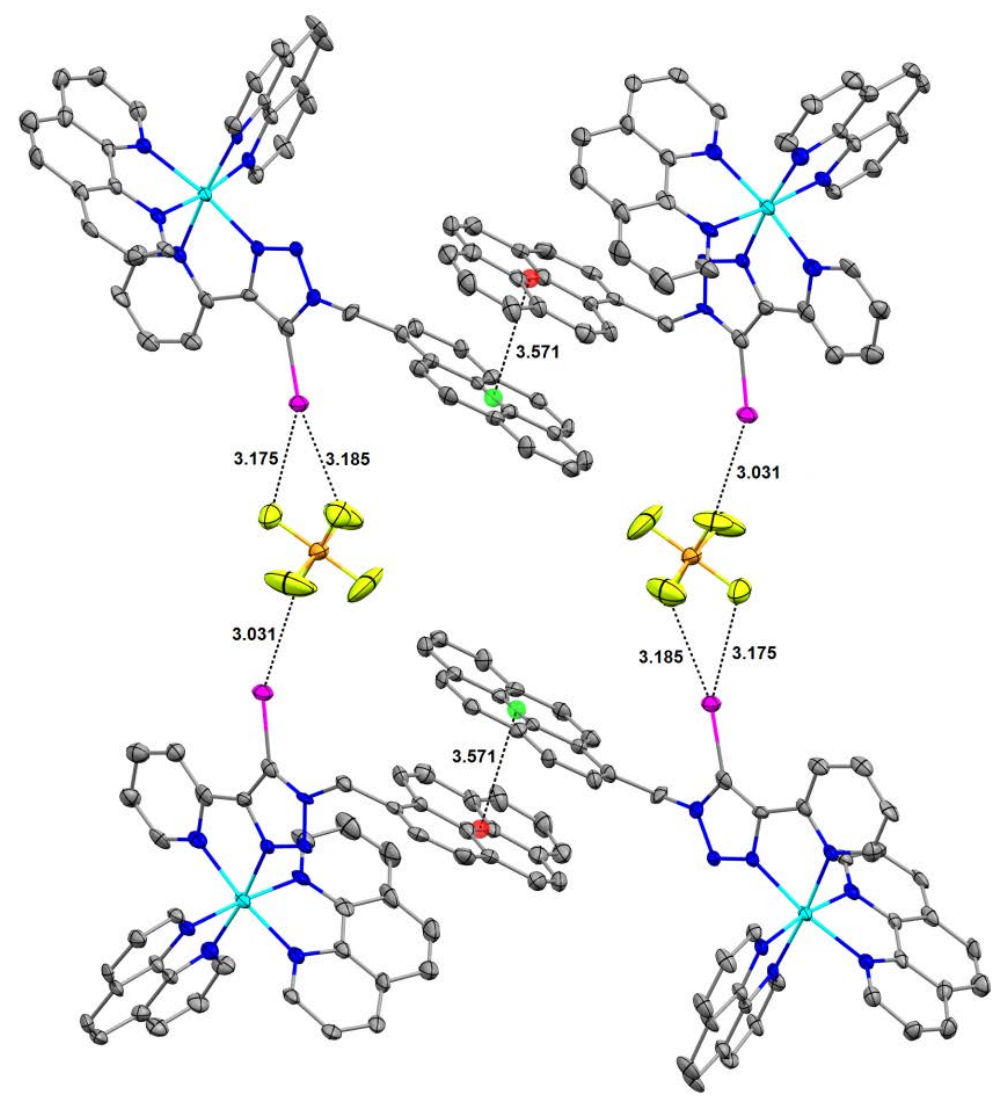

Figure S11: Thermal ellipsoid plot of $\mathbf{1}\left[\mathbf{P F}_{\mathbf{6}}\right]_{2}$. (a) Showing C-I...F interaction and (b) showing combined C-I $\cdots \mathrm{F}$ and $\pi-\pi$ stacking interaction. All Ellipsoids are drawn at $30 \%$ probability level. Color code: C, grey; Ru, cyan; N, blue; I, pink; F, yellowish green; P, orange yellow.

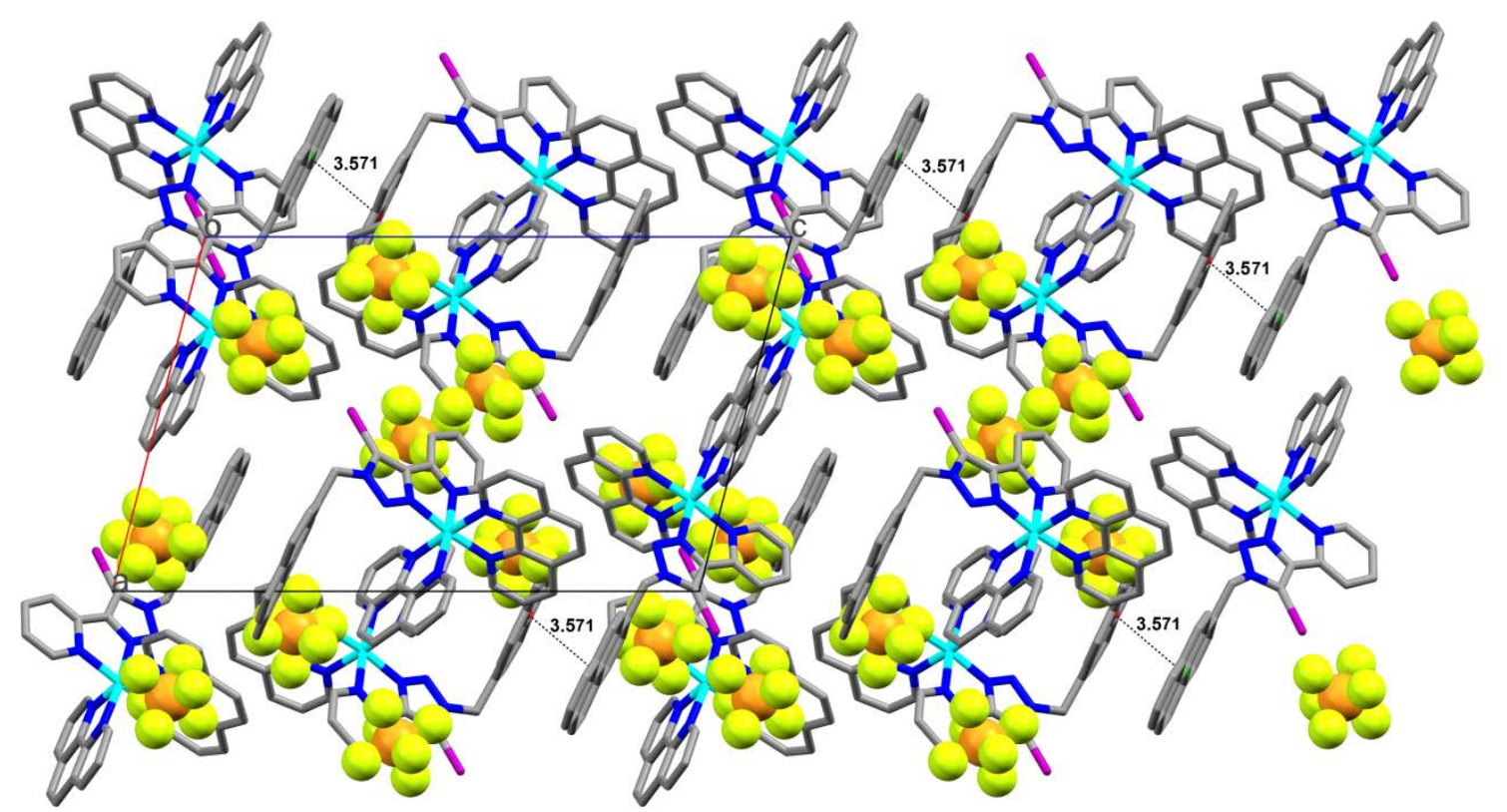

Figure S12: A slice of three-dimensional (3-D) crystal packing of $\mathbf{1}\left[\mathbf{P F}_{\mathbf{6}} \mathbf{l}_{2}\right.$ along the crystallographic b axis. Color code: $\mathrm{C}$, grey; Ru, cyan; N, blue; I, pink; F, yellowish green; P, orange yellow. 
(a)

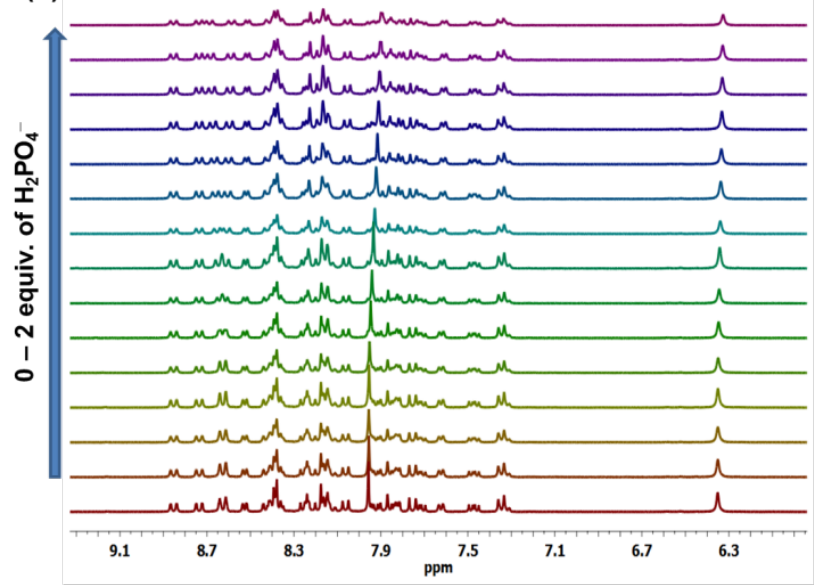

(b)

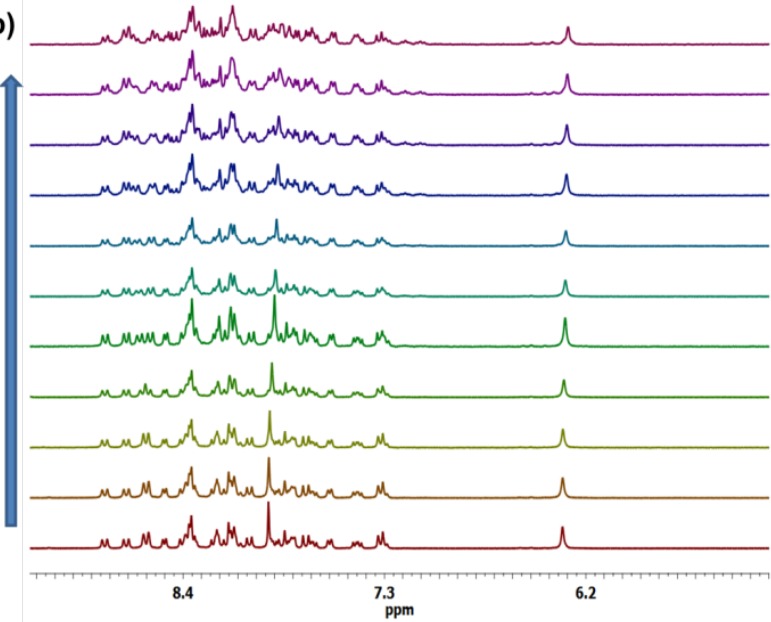

Figure S13: ${ }^{1} \mathrm{H}-\mathrm{NMR}$ titration profile of $\mathbf{1}\left[\mathbf{P F}_{\mathbf{6}} \mathbf{]}_{\mathbf{2}}\right.$ upon addition of increasing amount of (a) $\mathrm{H}_{2} \mathrm{PO}_{4}^{-}$(0-2.0 equiv) and (b) $\mathrm{HP}_{2} \mathrm{O}_{7}{ }^{3-}$ (0-2.0 equiv)

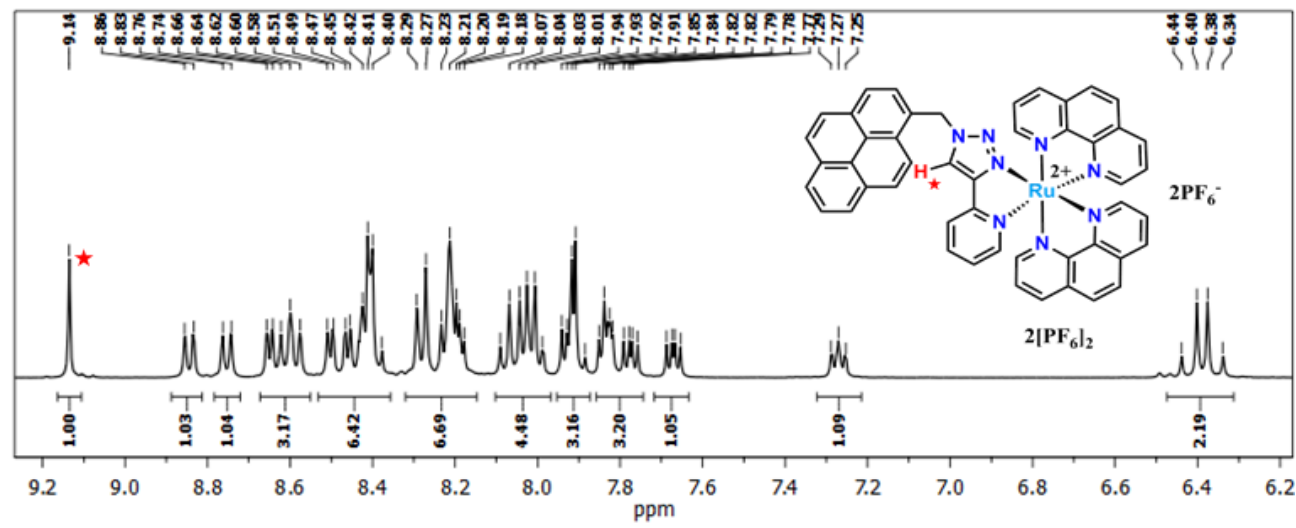

Figure S14: ${ }^{1} \mathrm{H}-\mathrm{NMR}(400 \mathrm{MHz})$ spectrum of control complex $\mathbf{2}\left[\mathbf{P} \mathbf{F}_{\mathbf{6}}\right]_{\mathbf{2}}$ in Acetone- $d_{6}$.
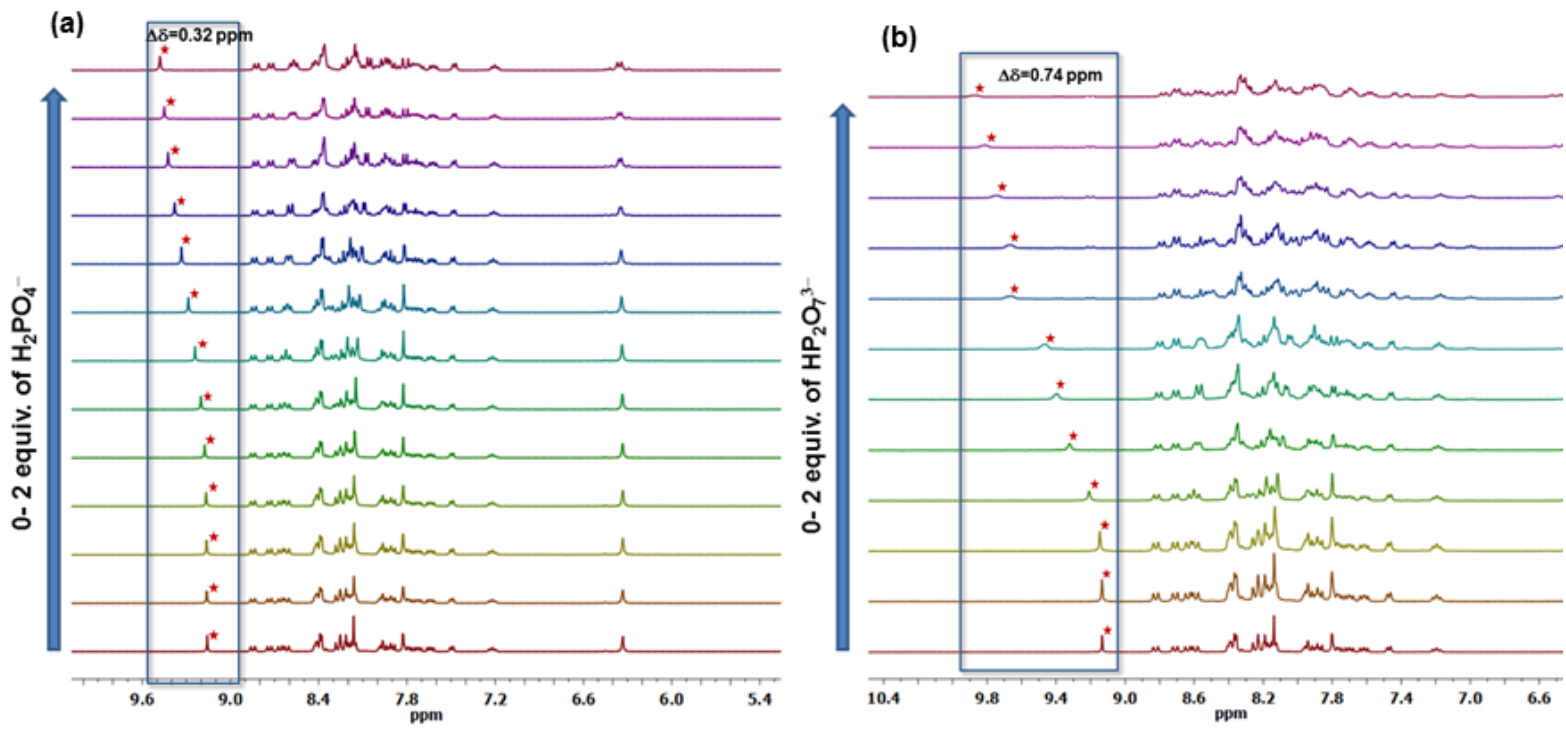

Figure S15: ${ }^{1} \mathrm{H}-\mathrm{NMR}$ titration profile of control complex $\mathbf{2}\left[\mathbf{P F}_{\mathbf{6}} \mathbf{]}_{\mathbf{2}}\right.$ upon addition of increasing amount of (a) $\mathrm{H}_{2} \mathrm{PO}_{4}{ }^{-}$(0-2.0 equiv) and (b) $\mathrm{HP}_{2} \mathrm{O}_{7}{ }^{3-}$ (0-2.0 equiv) respectively in DMSO- $d_{6}$. 
(a) uduldowhed

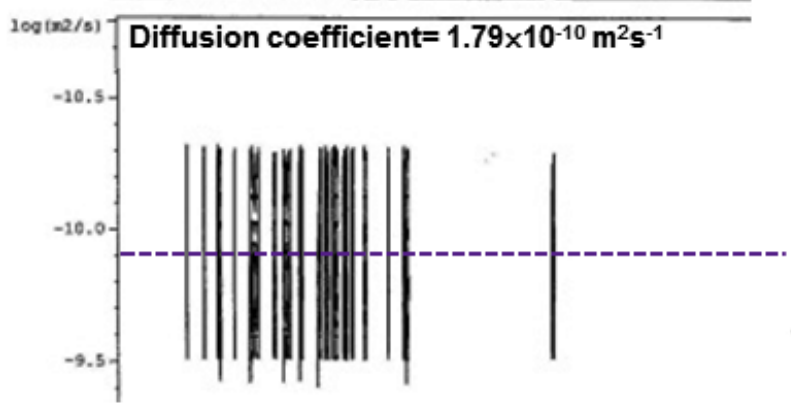

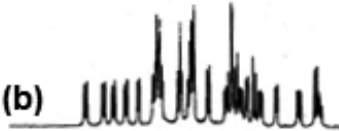

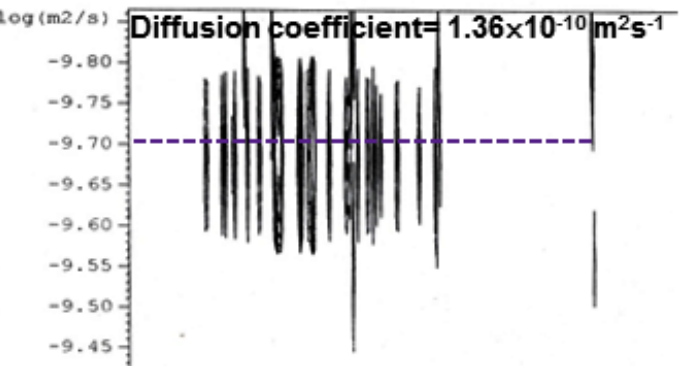

(c) cum dididual

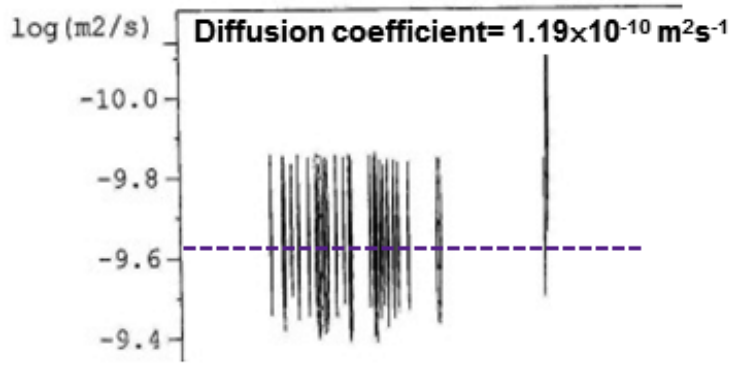

Figure S16: ${ }^{1} \mathrm{H}$ DOSY-NMR (500 MHz) spectrum of (a) $\mathbf{1}\left[\mathbf{P F}_{\mathbf{6}}\right]_{\mathbf{2}}$; (b) $\mathbf{1}\left[\mathbf{P F}_{\mathbf{6}}\right]_{\mathbf{2}}+\mathrm{HP}_{2} \mathrm{O}_{7}{ }^{3-}$ and (c) $\mathbf{1}\left[\mathbf{P F}_{6}\right]_{2}+\mathrm{H}_{2} \mathrm{PO}_{4}^{-}(10 \mathrm{mM})$ in DMSO- $d_{6}$ at $298 \mathrm{~K}$.
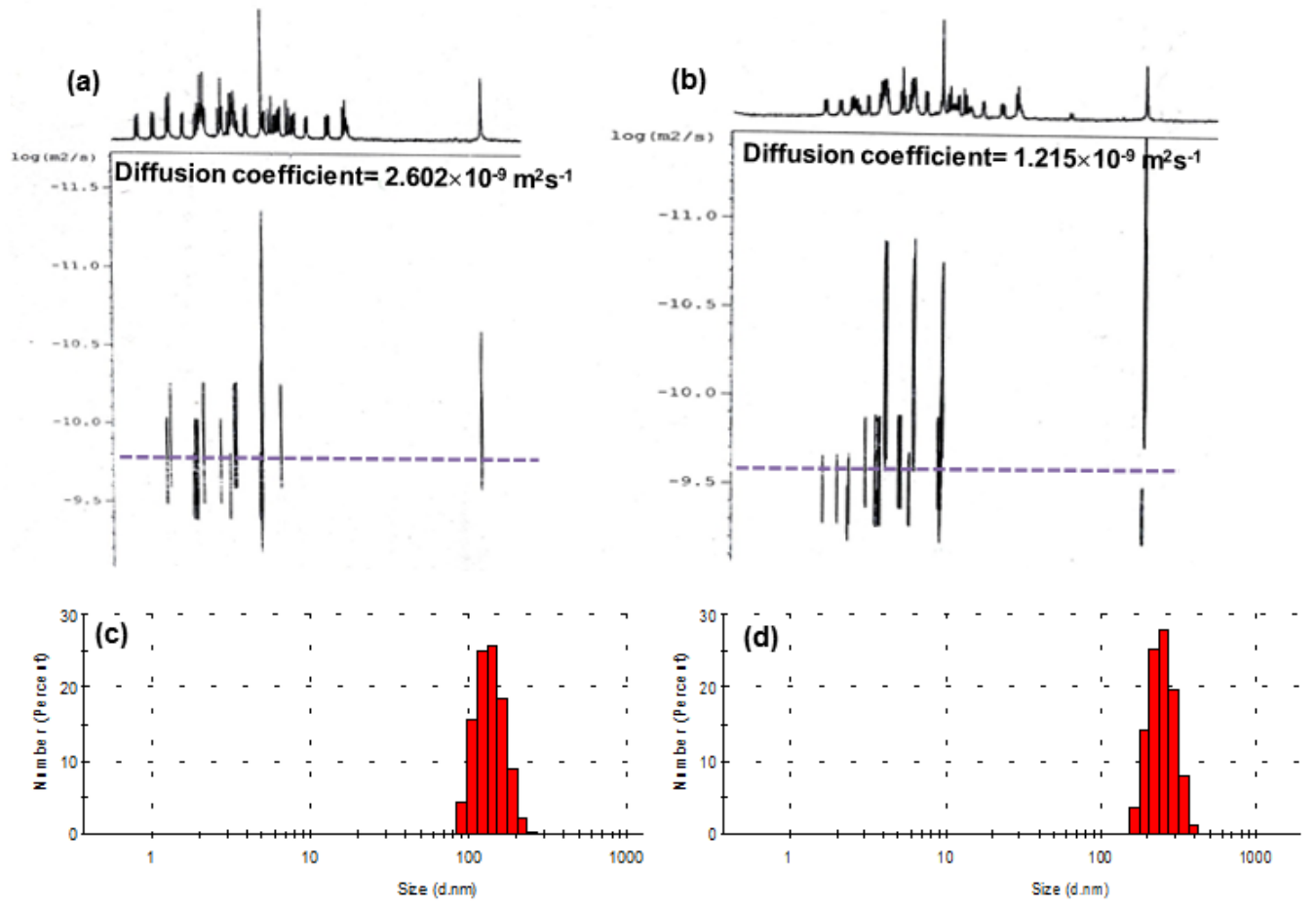

Figure S17: ${ }^{1} \mathrm{H}$ DOSY-NMR (500 MHz) spectrum of (a) $\mathbf{1}\left[\mathbf{P F}_{\mathbf{6}} \mathbf{]}_{\mathbf{2}}\right.$, (b) $\mathbf{1}\left[\mathbf{P F}_{\mathbf{6}} \mathbf{l}_{\mathbf{2}}+\mathrm{H}_{2} \mathrm{PO}_{4}{ }^{-}(10 \mu \mathrm{M})\right.$ in DMSO- $d_{6}$ at $298 \mathrm{~K}$ and DLS experiment of (c) $\mathbf{1}\left[\mathbf{P F}_{\mathbf{6}} \mathbf{]}_{\mathbf{2}}\right.$, (d) $\mathbf{1}\left[\mathbf{P F}_{\mathbf{6}} \mathbf{]}_{\mathbf{2}}+\mathrm{H}_{2} \mathrm{PO}_{4}{ }^{-}(10 \mu \mathrm{M})\right.$ in DMSO. 
Table S3: Calculation of sphere volume ratio from DOSY and DLS experiments.

\begin{tabular}{|c|c|c|c|c|c|c|}
\hline \multirow[t]{2}{*}{ Entry } & \multirow{2}{*}{$\begin{array}{l}\text { Complex } \\
(10 \mu \mathrm{M})\end{array}$} & \multicolumn{3}{|c|}{ DOSY Experiment data } & \multicolumn{2}{|c|}{$\begin{array}{c}\text { DLS Experiment } \\
\text { data }\end{array}$} \\
\hline & & $\begin{array}{l}\text { Diffusion } \\
\text { coefficient } \\
\mathrm{D}\left(\mathrm{m}^{2} / \mathrm{S}^{-1}\right)\end{array}$ & $\begin{array}{l}\text { Hydrodyn } \\
\text { amic } \\
\text { radius(nm) }\end{array}$ & $\begin{array}{l}\text { Sphere } \\
\text { volume } \\
\text { ratio }(\mathrm{B} / \mathrm{A})\end{array}$ & $\begin{array}{l}\text { Size of } \\
\text { particle } \\
(\mathrm{d} \mathrm{nm})\end{array}$ & $\begin{array}{l}\text { Sphere } \\
\text { volume } \\
\text { ratio(B/A) }\end{array}$ \\
\hline A & $1\left[\mathrm{PF}_{6}\right]_{2}$ & $2.602 \times 10^{-9}$ & 0.04 & \multirow[t]{2}{*}{9} & 140 & \multirow[t]{2}{*}{7.9} \\
\hline $\mathrm{B}$ & $1\left[\mathrm{PF}_{6}\right]_{2}+\mathrm{H}_{2} \mathrm{PO}_{4}^{-}$ & $1.215 \times 10^{-9}$ & 0.08 & & 280 & \\
\hline
\end{tabular}

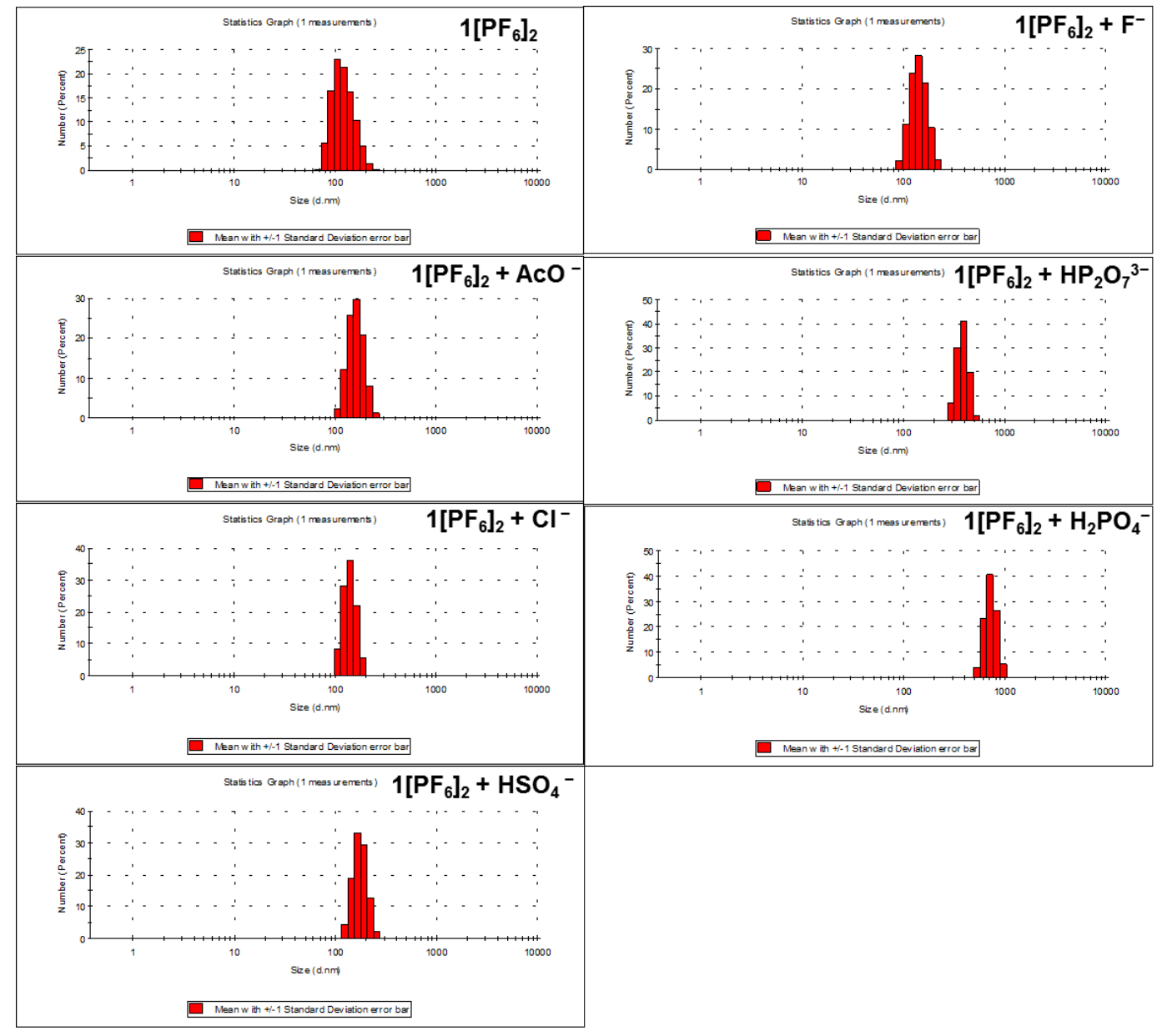

Figure S18: Anion dependent DLS experiment in DMSO at 298K. 


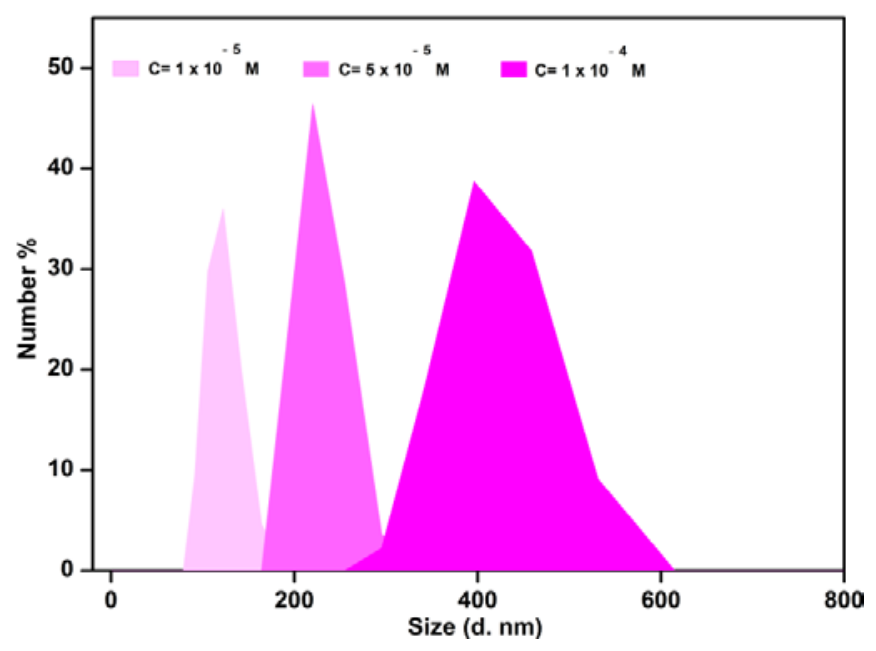

Figure S19: Concentration dependent DLS study of $\mathbf{1}\left[\mathbf{P F}_{\mathbf{6}}\right]_{2}$ in presence of $\mathrm{HP}_{2} \mathrm{O}_{7}{ }^{3-}$ adduct.

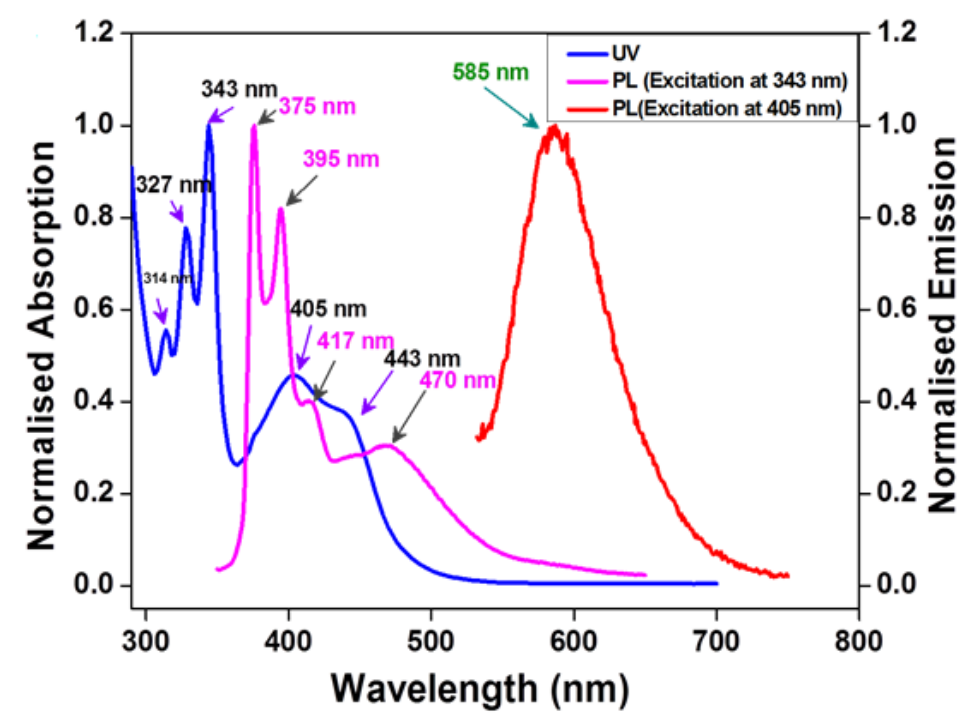

Figure S20: UV and PL plot of $\mathbf{1}\left[\mathbf{P F}_{\mathbf{6}}\right]_{2}$ in acetonitrile at $298 \mathrm{~K}$.

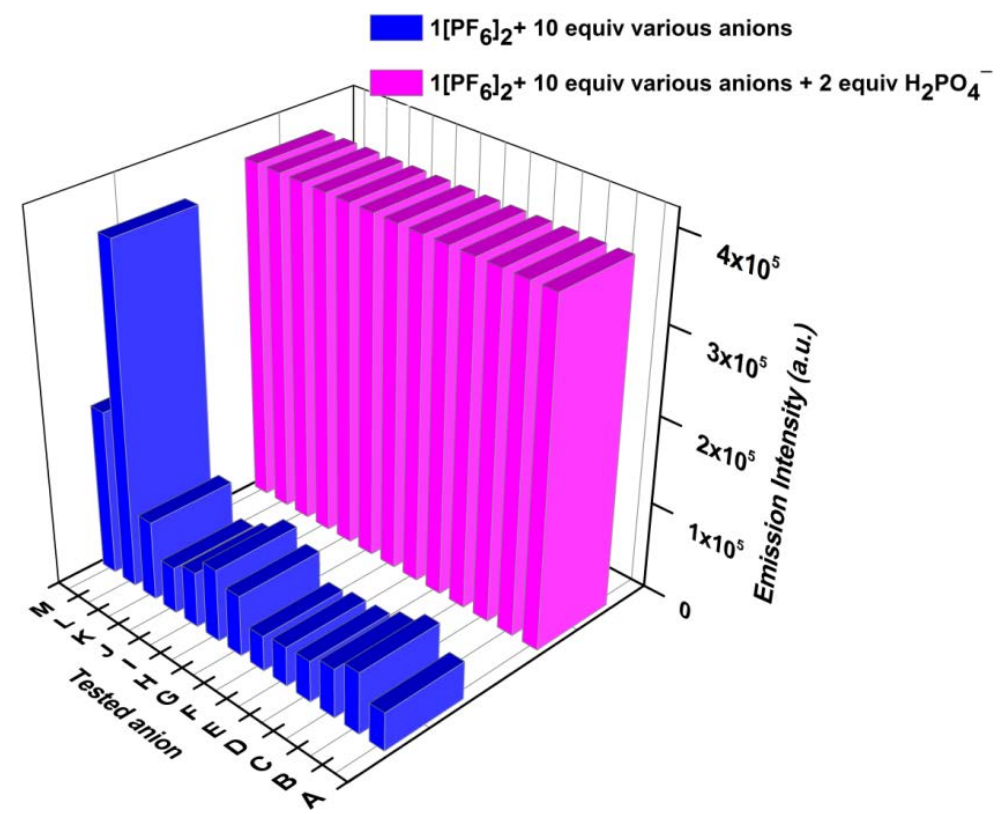


Figure S21: Selectivity bar diagram of $\mathbf{1}\left[\mathbf{P F}_{\mathbf{6}}\right]_{\mathbf{2}}$ in acetonitrile. Numbers code: (A) $\mathbf{1}\left[\mathbf{P F}_{\mathbf{6}}\right]_{2},(B)$ $\mathrm{Cl}^{-}$, (C) $\mathrm{Br}^{-}$, (D) $\mathrm{I}^{-}$,(E) $\mathrm{HSO}_{4}{ }^{-}$,(F) $\mathrm{NO}_{3}{ }^{-}$, (G) $\mathrm{F}^{-}$, (H) $\mathrm{AcO}^{-}$, (I) $\mathrm{BzO}^{-}$, (J) $\mathrm{HCO}_{3}{ }^{-}$, (K) $\mathrm{HO}^{-}$, (L) $\mathrm{H}_{2} \mathrm{PO}_{4}^{-}$. (M) $\mathrm{HP}_{2} \mathrm{O}_{7}^{3-}$.
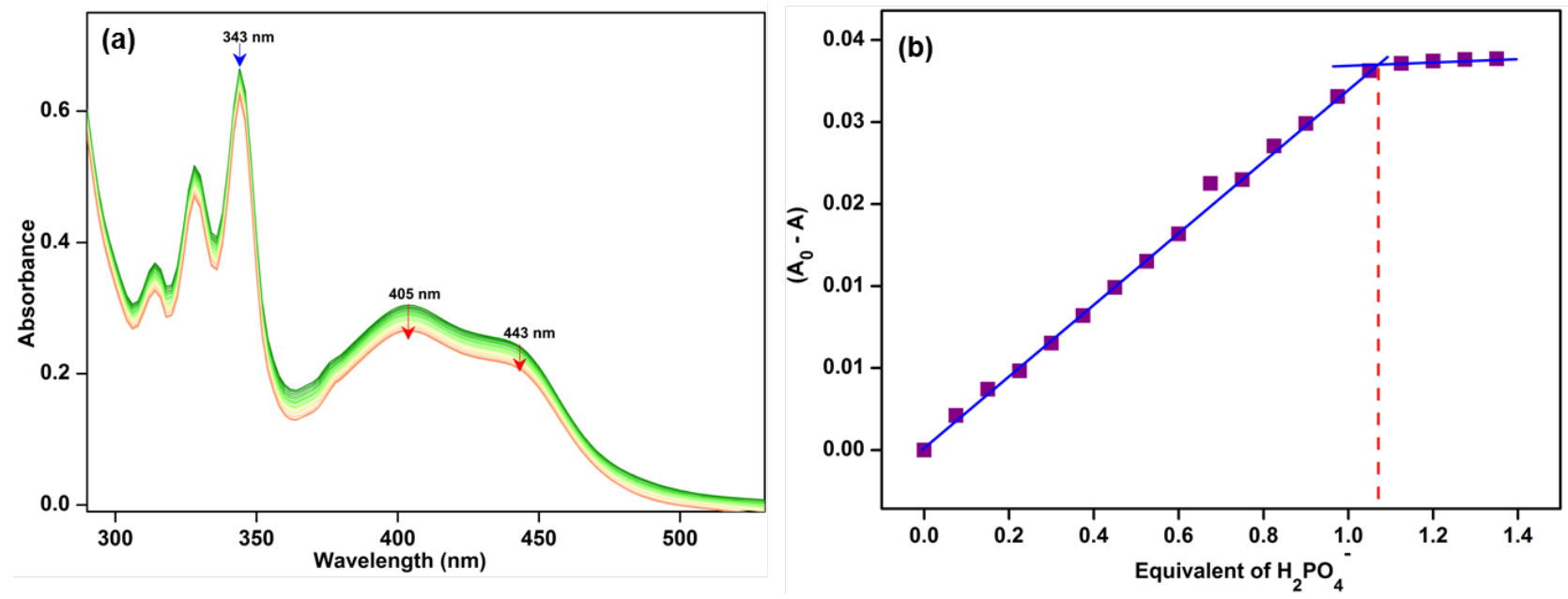

Figure S22: (a) UV-vis titration profile of $\mathbf{1}\left[\mathbf{P F}_{\mathbf{6}} \mathbf{]}_{2}(40 \mu \mathrm{M})\right.$ with $\mathrm{H}_{2} \mathrm{PO}_{4}{ }^{-}$(0-1.5 equiv) (b) Anion equivalent plots from UV-vis titration data of $\mathbf{1}\left[\mathbf{P F}_{6}\right]_{2}(40 \mu \mathrm{M})$ with $\mathrm{H}_{2} \mathrm{PO}_{4}{ }^{-}$(0-1.5 equiv) in acetonitrile at $298 \mathrm{~K}$.
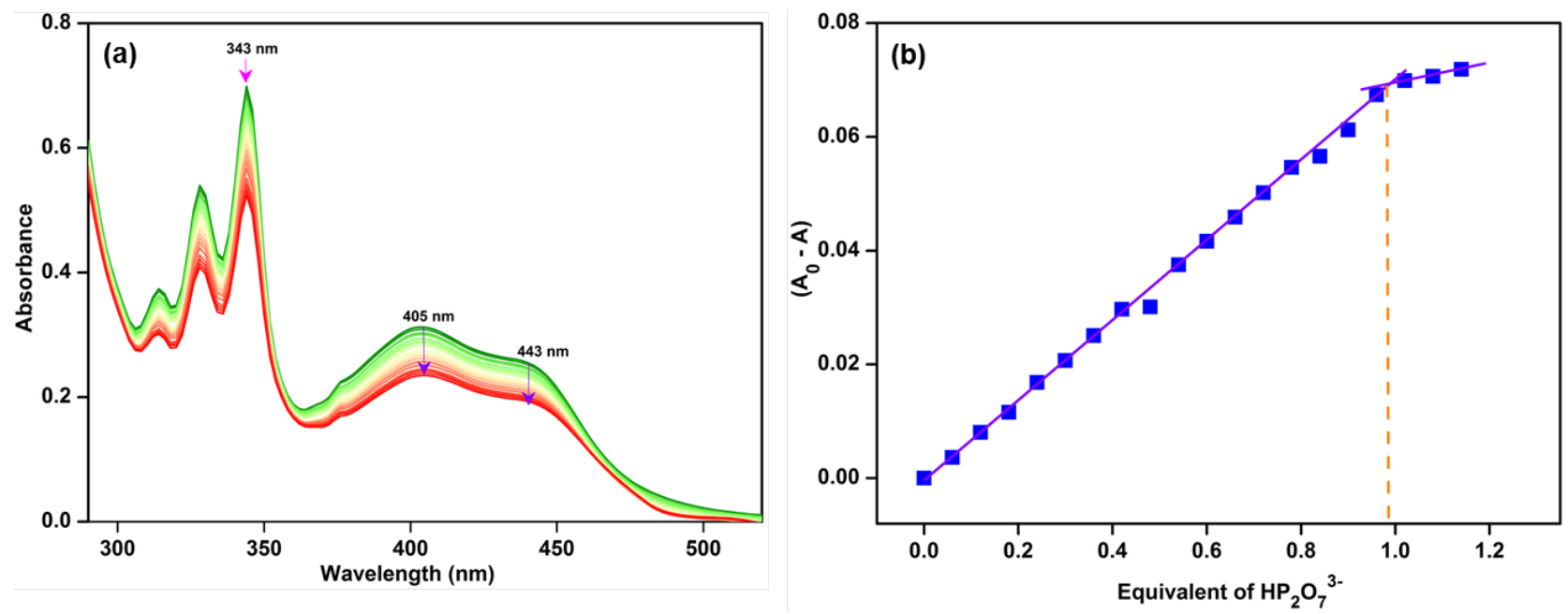

Figure S23: (a) UV-vis titration profile of $\mathbf{1}\left[\mathbf{P F}_{\mathbf{6}} \mathbf{]}_{\mathbf{2}}(40 \mu \mathrm{M})\right.$ with $\mathrm{HP}_{2} \mathrm{O}_{7}{ }^{3-}$ (0-1.5 equiv) (b) Anion equivalent plots from UV-vis titration data of $\mathbf{1}\left[\mathbf{P F}_{\mathbf{6}} \mathbf{]}_{2}(40 \mu \mathrm{M})\right.$ with $\mathrm{HP}_{2} \mathrm{O}_{7}{ }^{3-}$ (0-1.5 equiv) in acetonitrile at $298 \mathrm{~K}$. 

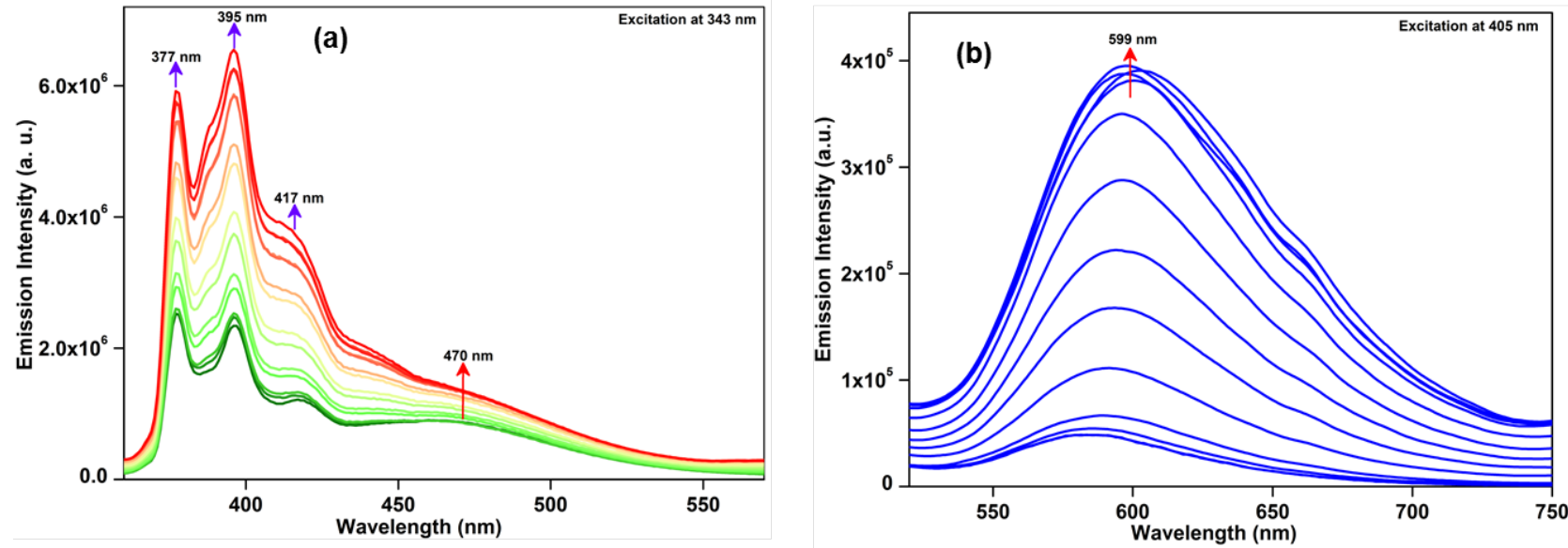

Figure S24: PL titration profile of $\mathbf{1}\left[\mathbf{P F}_{\mathbf{6}}\right]_{2}(10 \mu \mathrm{M})$ with $\mathrm{H}_{2} \mathrm{PO}_{4}{ }^{-}(0-1.5$ equiv) in acetonitrile at 298K.
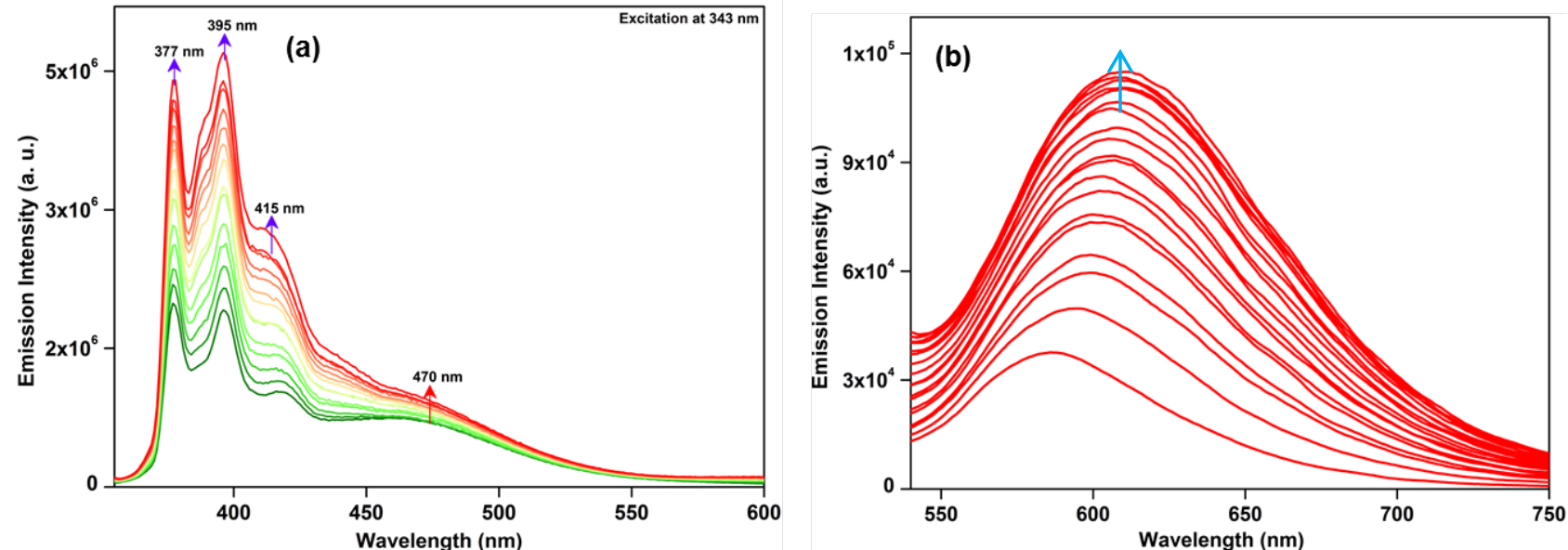

Figure S25: PL titration profile of $\mathbf{1}\left[\mathbf{P F}_{\mathbf{6}}\right]_{2}(10 \mu \mathrm{M})$ with $\mathrm{HP}_{2} \mathrm{O}_{7}{ }^{3-}(0-1.5$ equiv) in acetonitrile at 298K.
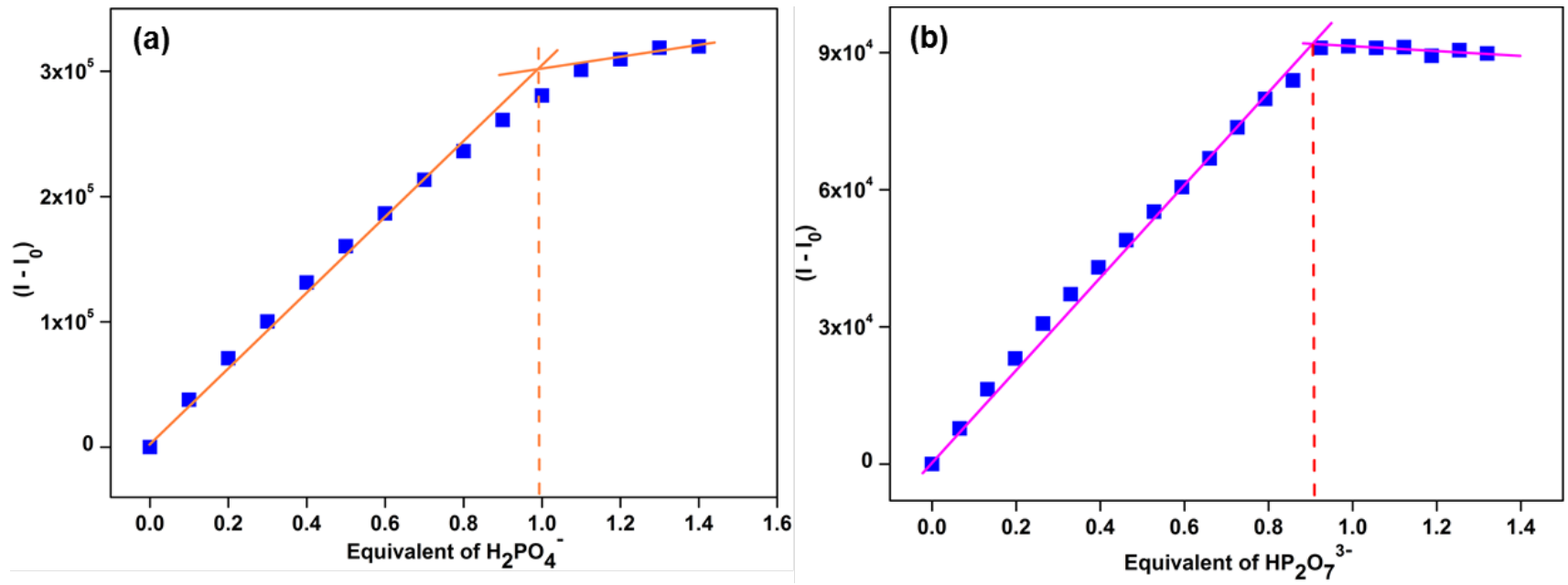

Figure S26: Anion equiv. plot from PL titration profile of $\mathbf{1}\left[\mathbf{P F}_{\mathbf{6}} \mathbf{l}_{2}(10 \mu \mathrm{M})\right.$ with (a) $\mathrm{H}_{2} \mathrm{PO}_{4}{ }^{-}(0-$ 1.5 equiv) and (b) $\mathrm{HP}_{2} \mathrm{O}_{7}{ }^{3-}$ (0-1.5 equiv) in acetonitrile at $298 \mathrm{~K}$. 


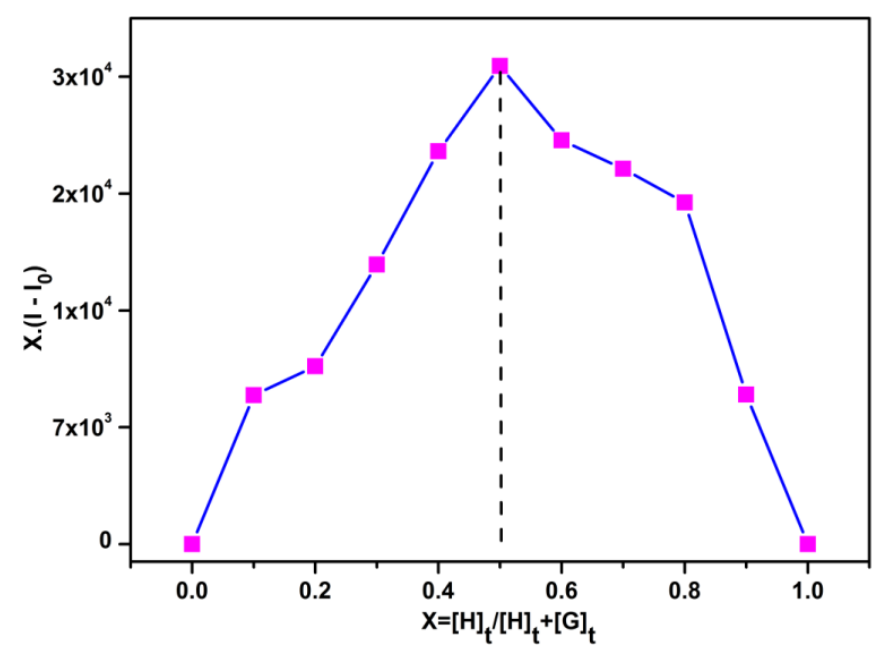

Figure S27: Job's plots from PL of $\mathbf{1}\left[\mathbf{P F}_{6}\right]_{2}(20 \mu \mathrm{M})$ with $\mathrm{H}_{2} \mathrm{PO}_{4}{ }^{-}(20 \mu \mathrm{M})$ in acetonitrile at 298K.

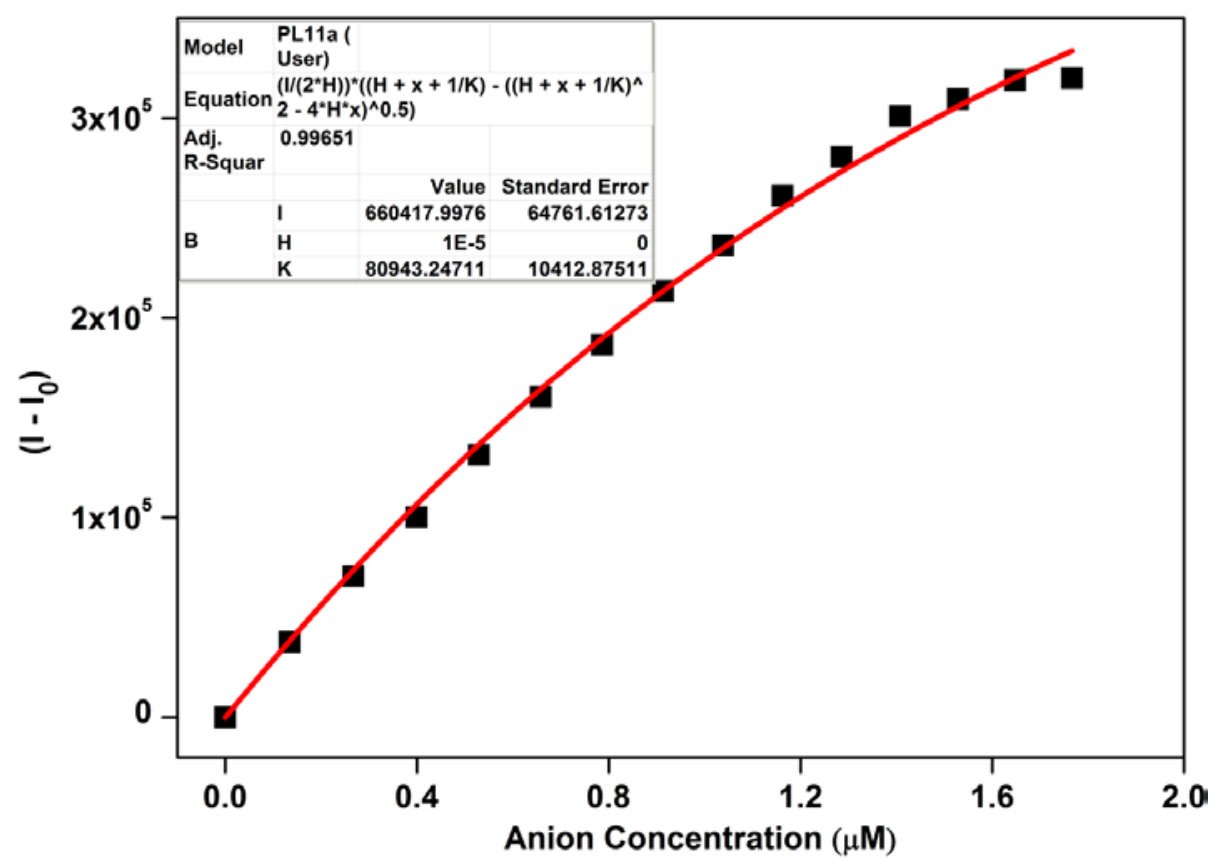

Figure S28: Non-linear 1:1 fitting of PL titration data to calculate association constant of $\mathbf{1}\left[\mathbf{P F}_{6}\right]_{2}$ with $\mathrm{H}_{2} \mathrm{PO}_{4}{ }^{-}$. 


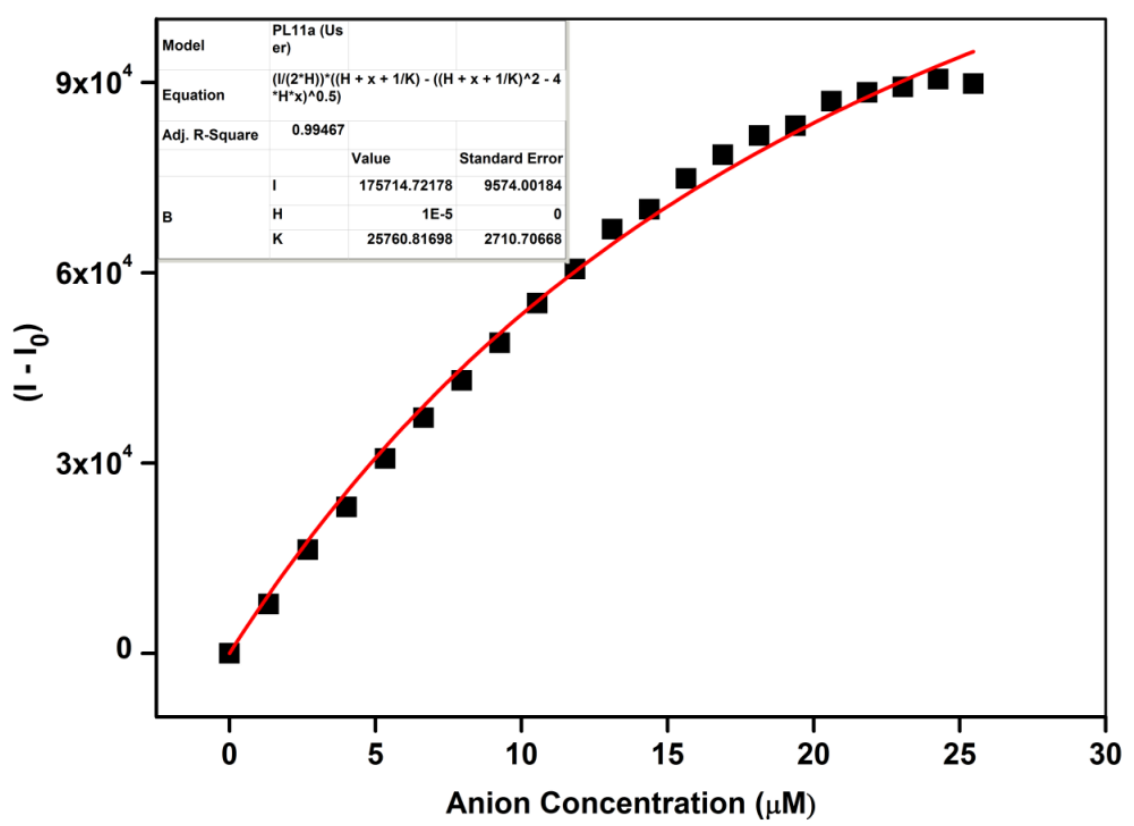

Figure S29: Non-linear 1:1 fitting of PL titration data to calculate association constant of $\mathbf{1}\left[\mathbf{P F}_{6}\right]_{2}$ with $\mathrm{HP}_{2} \mathrm{O}_{7}{ }^{3-}$

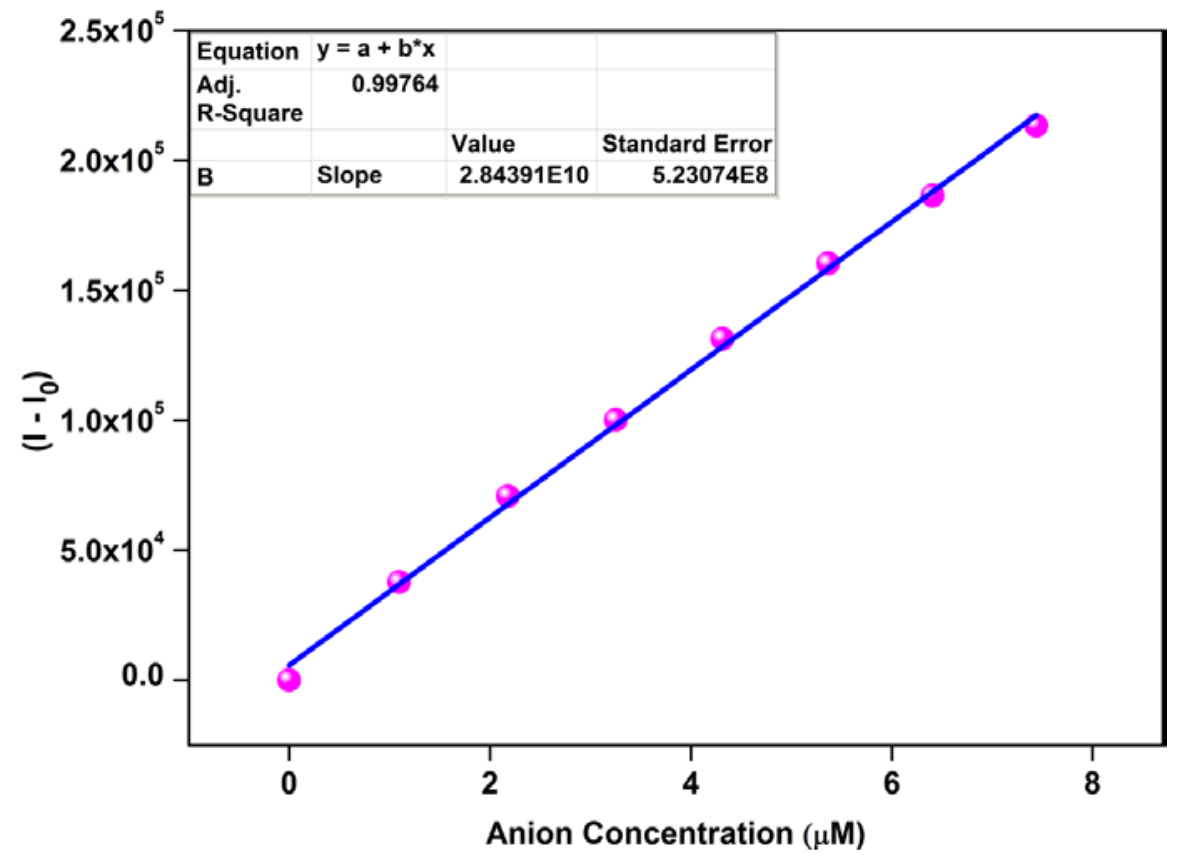

Figure S30: Calibration curve for $\mathrm{H}_{2} \mathrm{PO}_{4}{ }^{-}$with probe $\mathbf{1}\left[\mathbf{P F}_{\mathbf{6}}\right]_{2}$ for the calculation of detection limit in acetonitrile at $298 \mathrm{~K}$. 


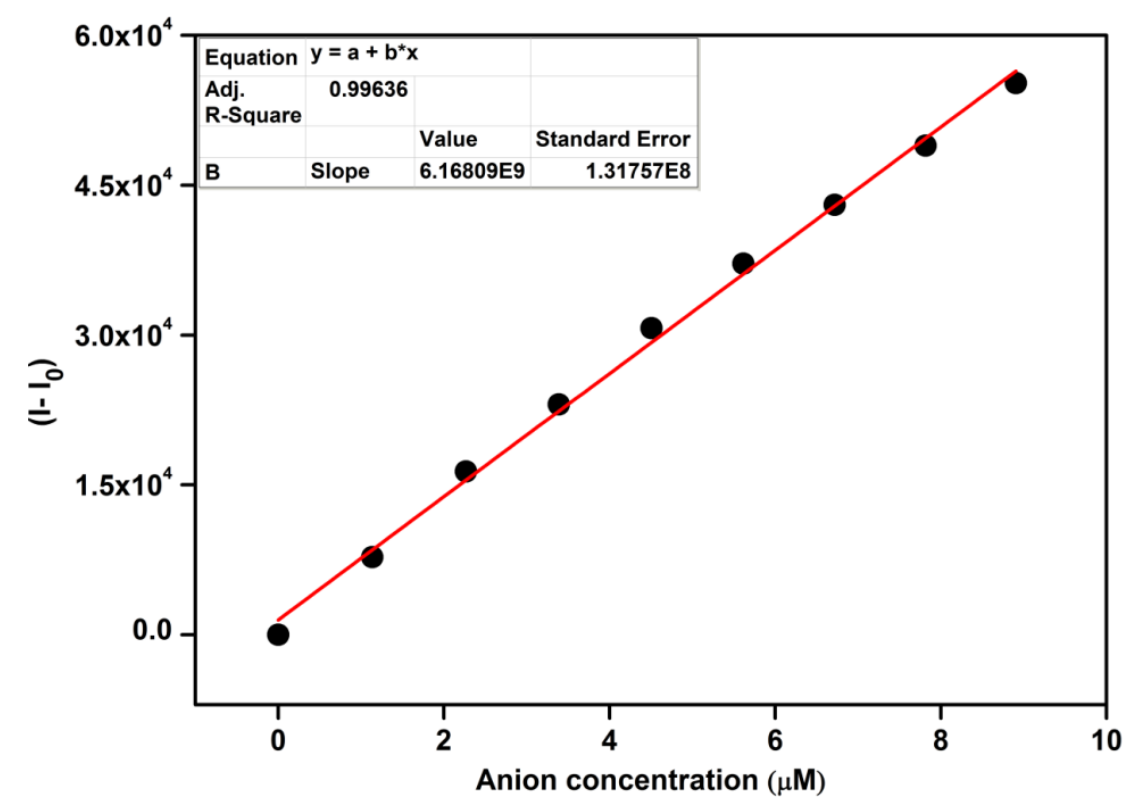

Figure S31: Calibration curve for $\mathrm{HP}_{2} \mathrm{O}_{7}{ }^{3-}$ with probe $\mathbf{1}\left[\mathbf{P F}_{\mathbf{6}}\right]_{\mathbf{2}}$ for the calculation of detection limit in acetonitrile at $298 \mathrm{~K}$.

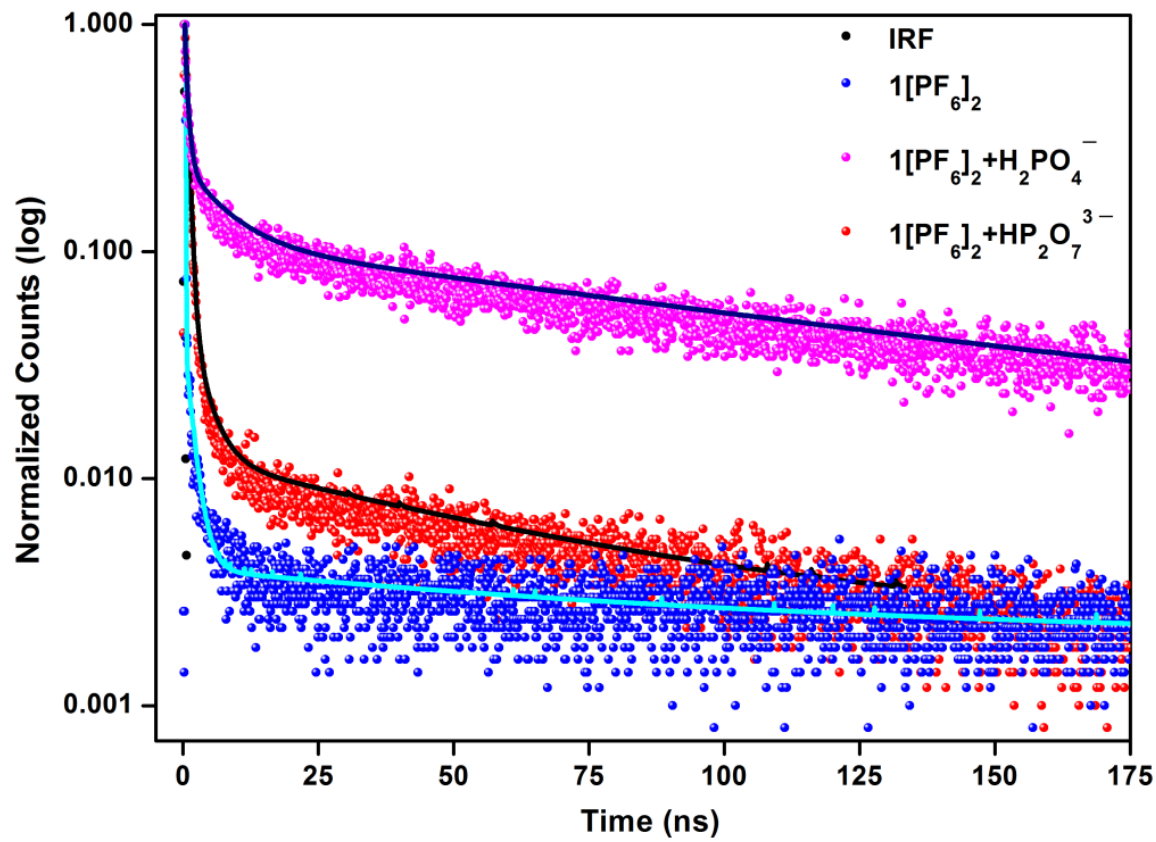

Figure S32: Changes in decay pattern of $\mathbf{1}\left[\mathbf{P F}_{6}\right]_{2}(10 \mu \mathrm{M})$ and upon addition of one eqv. of $\mathrm{H}_{2} \mathrm{PO}_{4}{ }^{-}$and $\mathrm{HP}_{2} \mathrm{O}_{7}{ }^{3-}$. 


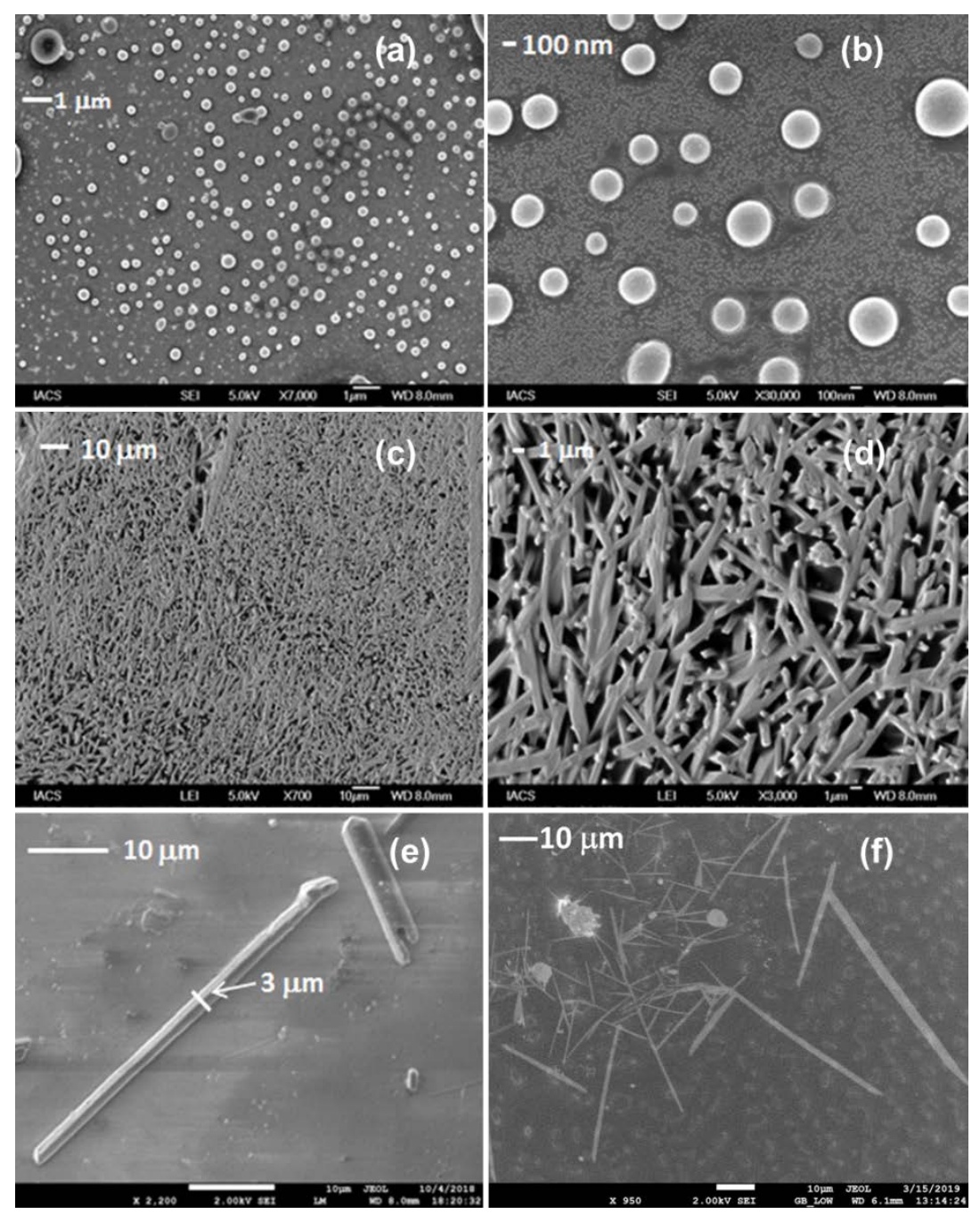

Figure S33: FESEM images of $\mathbf{1}\left[\mathbf{P F}_{\mathbf{6}}\right]_{\mathbf{2}}(\mathrm{a}, \mathrm{b})$ shows vesicular type morphology where as in presence of $\mathrm{H}_{2} \mathrm{PO}_{4}{ }^{-}$and $\mathrm{HP}_{2} \mathrm{O}_{7}{ }^{3-}$ shows the formation of linear rod like morphology (c, d, e and $\mathrm{f}$ respectively)

Table S4: Selected bond distances and bond angles of complex $\mathbf{1}\left[\mathbf{H}_{2} \mathbf{P O}_{4}\right]_{2}$.

\begin{tabular}{|c|c|c|c|c|c|}
\hline M-L & d(M-L)Å & L-M-L & $\angle(\mathrm{L}-\mathrm{M}-\mathrm{L})\left[^{\circ}\right]$ & L-M-L & $\angle(\mathrm{L}-\mathrm{M}-\mathrm{L})\left[^{\circ}\right]$ \\
\hline I1- COOV & $2.046(18)$ & N1-Ru1-N2 & $81.0(6)$ & N2-Ru1-N6 & $95.6(5)$ \\
\hline Ru1-N1 & $2.065(14)$ & N1-Ru1-N3 & $173.2(5)$ & N3-Ru1-N4 & $78.3(5)$ \\
\hline Ru1-N2 & $2.073(14)$ & N1-Ru1-N4 & $95.7(5)$ & N3-Ru1-N5 & $90.2(5)$ \\
\hline Ru1-N3 & $2.029(12)$ & N1-Ru1-N5 & $93.8(5)$ & N3-Ru1-N6 & $98.7(5)$ \\
\hline Ru1-N4 & $2.075(14)$ & N1-Ru1-N6 & $87.5(5)$ & N4-Ru1-N5 & 97.0(5) \\
\hline Ru1-N5 & $2.038(12)$ & N2-Ru1-N3 & $95.3(6)$ & N4-Ru1-N6 & 176.1(5) \\
\hline \multirow[t]{2}{*}{ Ru1-N6 } & \multirow[t]{2}{*}{$2.071(12)$} & N2-Ru1-N4 & $87.2(5)$ & N5-Ru1-N6 & $80.5(5)$ \\
\hline & & N2-Ru1-N5 & 173.7(5) & & \\
\hline
\end{tabular}


(a)

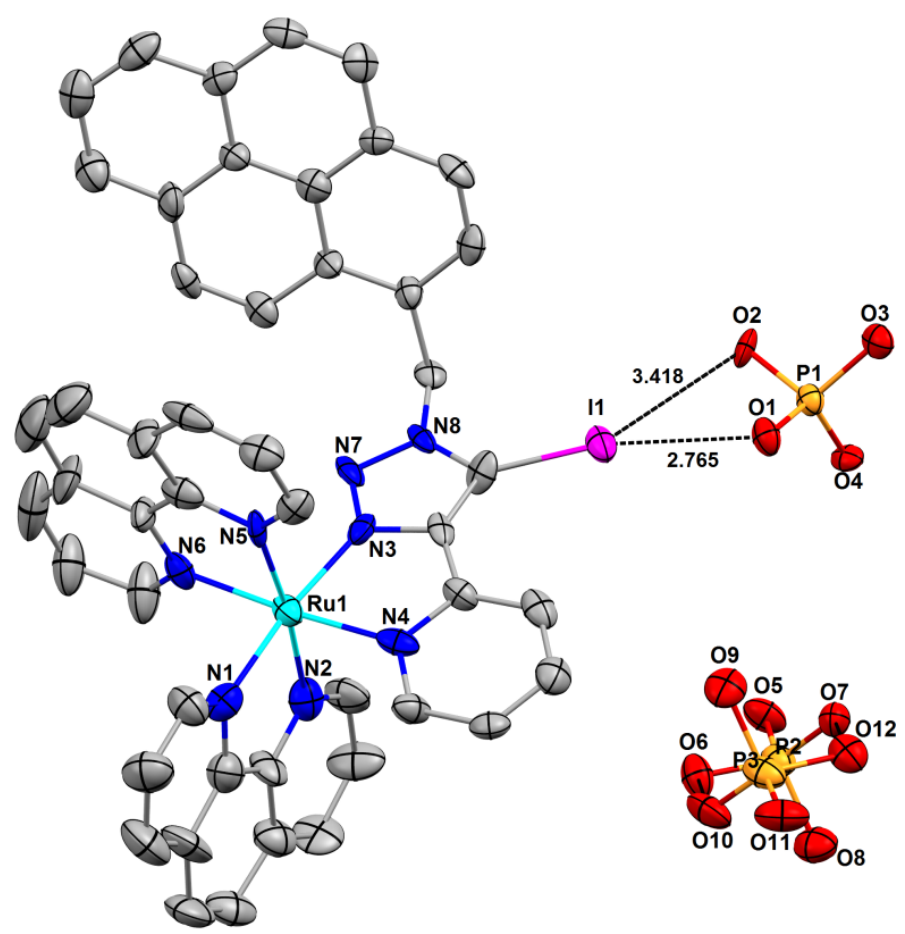

(b)

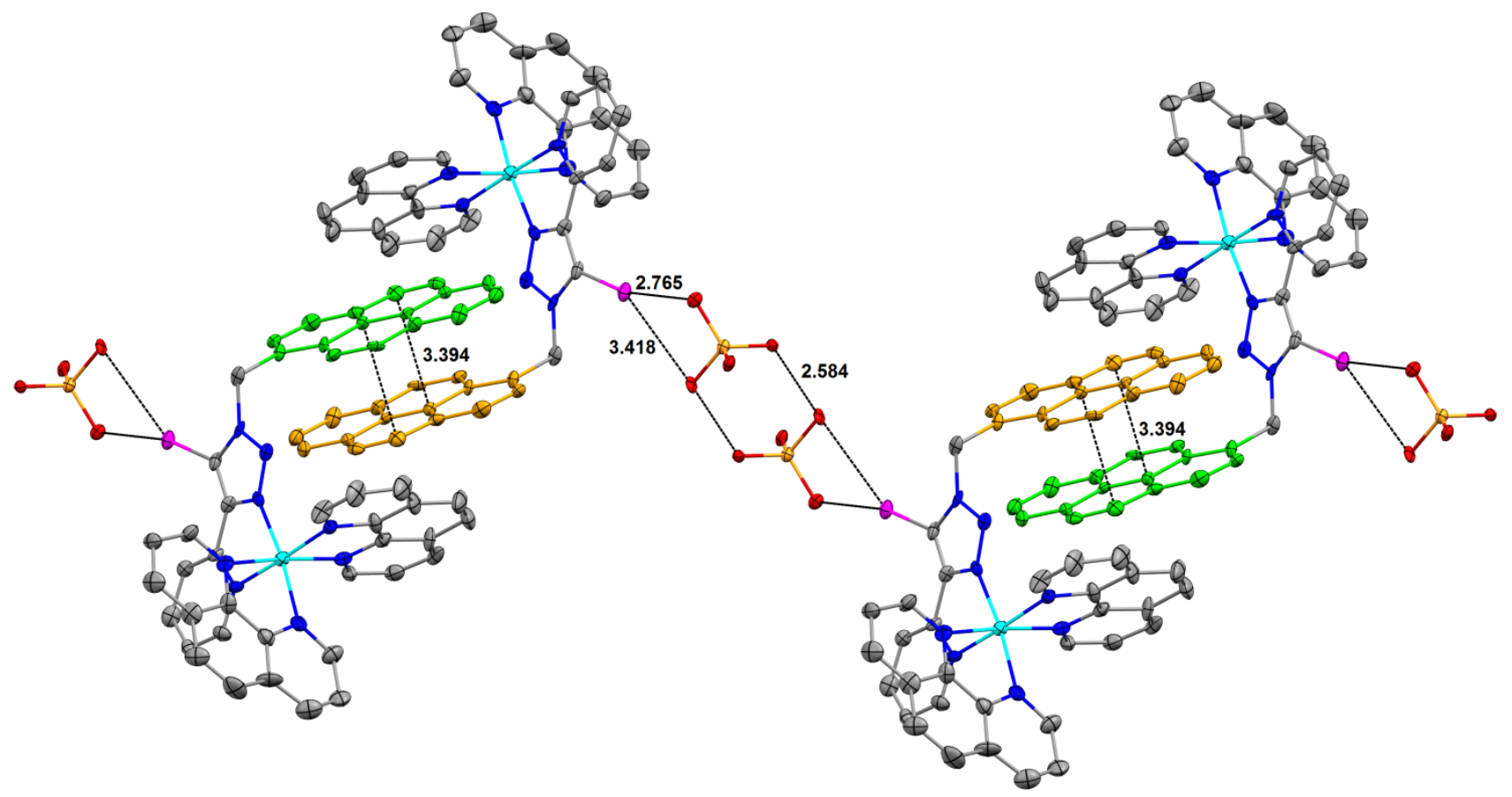

Figure S34: Thermal ellipsoid plot of $\mathbf{1}\left[\mathbf{H}_{2} \mathbf{P O}_{4}\right]_{2}$. (a) Showing C-I...O interaction and (b) showing combined $\mathrm{C}-\mathrm{I} \cdots \mathrm{O}$ and $\pi-\pi$ stacking interaction. All Ellipsoids are drawn at $30 \%$ probability level. Color code: C, grey, green, yellow; Ru, cyan; N, blue; I, pink; O, red; P, orange yellow. 
(a)

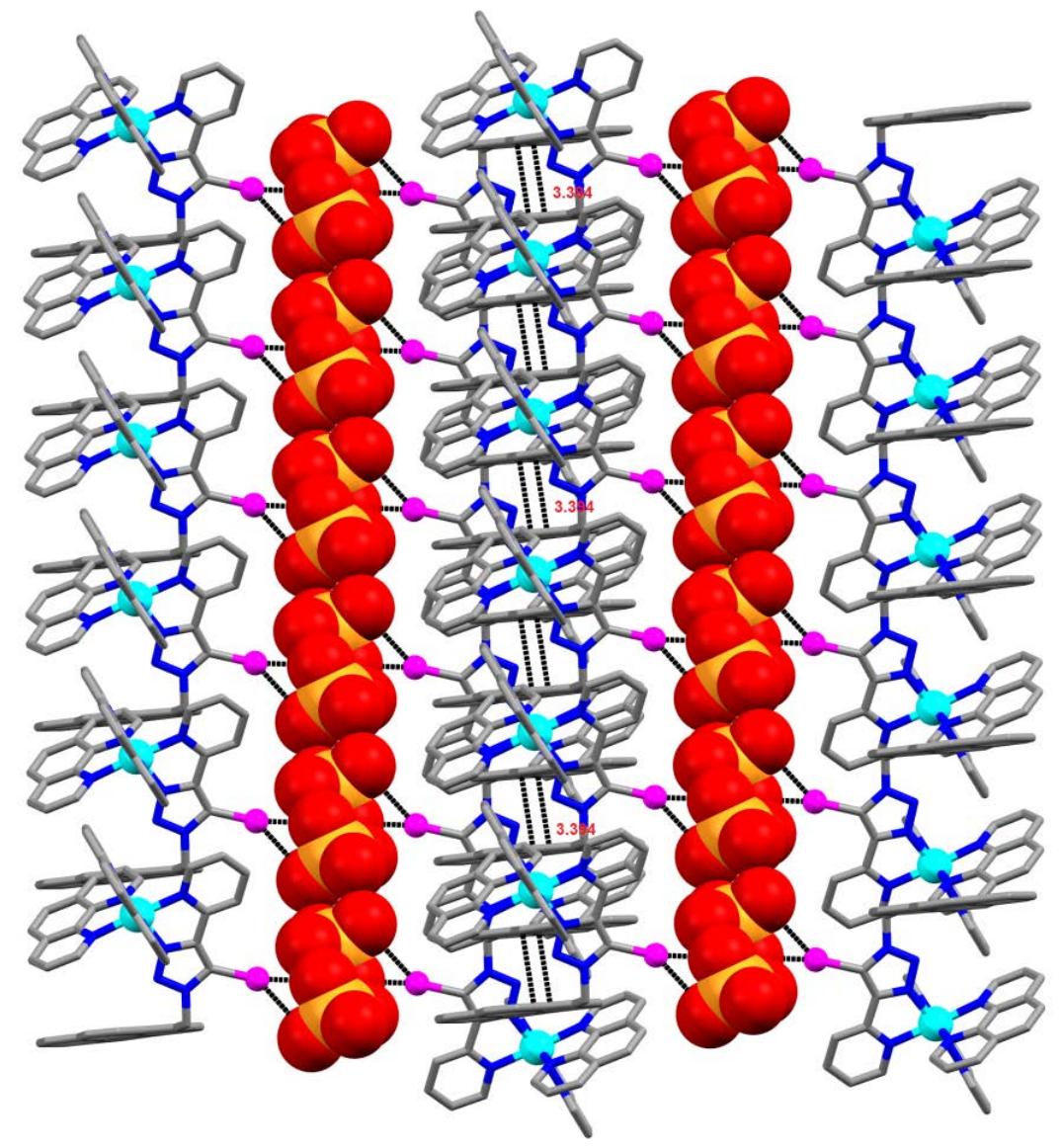

(b)

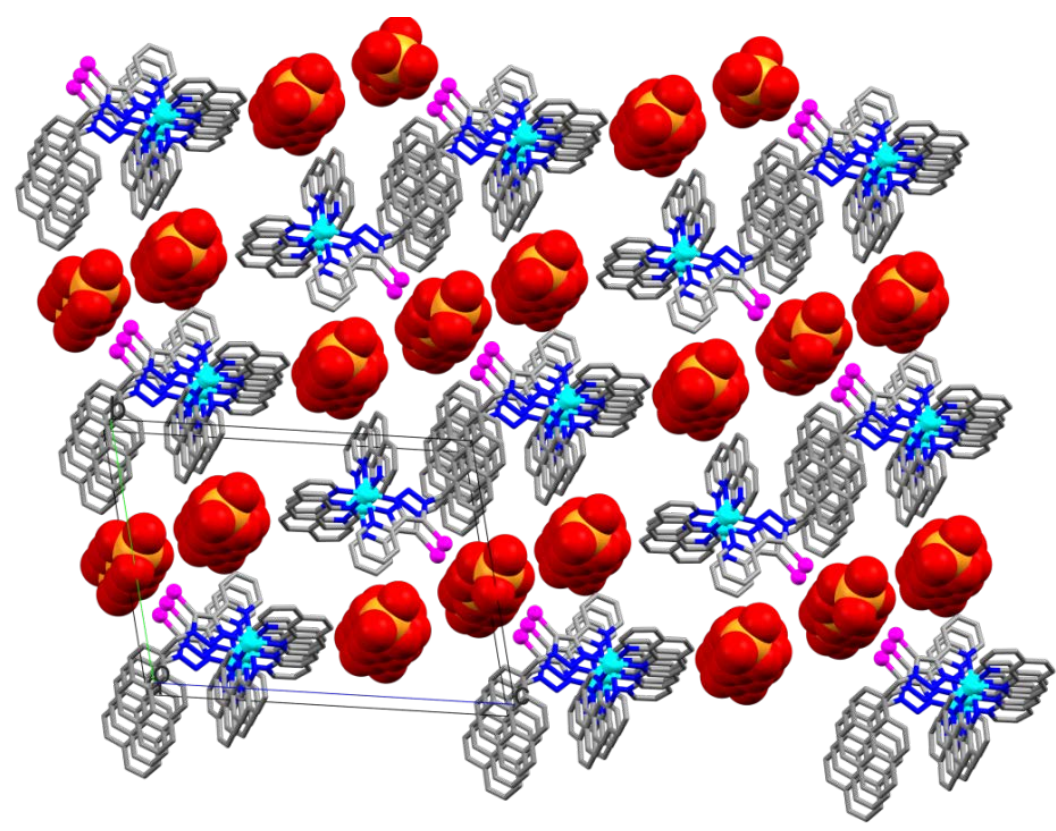

Figure S35: (a) Single-crystal X-ray structures of $\mathbf{1}\left[\mathbf{H}_{2} \mathbf{P O}_{4}\right]_{2}$ propagated through combined halogen bonding and $\pi-\pi$ stacking interaction. (b) A slice of three-dimensional (3-D) crystal packing of $\mathbf{1}\left[\mathbf{H}_{2} \mathbf{P O}_{4}\right]_{2}$ along the crystallographic a* axis. Color code: $\mathrm{C}$, grey; Ru, cyan; $\mathrm{N}$, blue; I, pink; O, red; P, orange yellow. 